\author{
Marquette University \\ e-Publications@Marquette
}

7-5-2017

\title{
MQCT. I. Inelastic Scattering of Two Asymmetric-Top Rotors with Application to $\mathrm{H}_{2} \mathrm{O}+\mathrm{H}_{2} \mathrm{O}$
}

\author{
Alexander Semenov \\ Marquette University \\ Dmitri Babikov \\ Marquette University, dmitri.babikov@marquette.edu
}

Follow this and additional works at: https://epublications.marquette.edu/chem_fac

Part of the Chemistry Commons

\section{Recommended Citation}

Semenov, Alexander and Babikov, Dmitri, "MQCT. I. Inelastic Scattering of Two Asymmetric-Top Rotors with Application to $\mathrm{H}_{2} \mathrm{O}+\mathrm{H}_{2} \mathrm{O}$ " (2017). Chemistry Faculty Research and Publications. 899.

https://epublications.marquette.edu/chem_fac/899 


\title{
Marquette University
}

\section{e-Publications@Marquette}

\section{Chemistry Faculty Research and Publications/College of Arts and Sciences}

This paper is NOT THE PUBLISHED VERSION; but the author's final, peer-reviewed manuscript. The published version may be accessed by following the link in th citation below.

Journal of Physical Chemistry: A, Vol. 121, No. 26 (2017): 4855-4867. DOI. This article is (C) American Chemical Society and permission has been granted for this version to appear in e-

Publications@Marquette. American Chemical Society does not grant permission for this article to be further copied/distributed or hosted elsewhere without the express permission from American Chemical Society.

\section{MQCT. I. Inelastic Scattering of Two Asymmetric-Top Rotors with Application to $\mathrm{H}_{2} \mathrm{O}+\mathrm{H}_{2} \mathrm{O}$}

\author{
Alexander Semenov \\ Chemistry Department, Wehr Chemistry Building, Marquette University, Milwaukee, WI \\ Dmitri Babikov \\ Chemistry Department, Wehr Chemistry Building, Marquette University, Milwaukee, WI
}

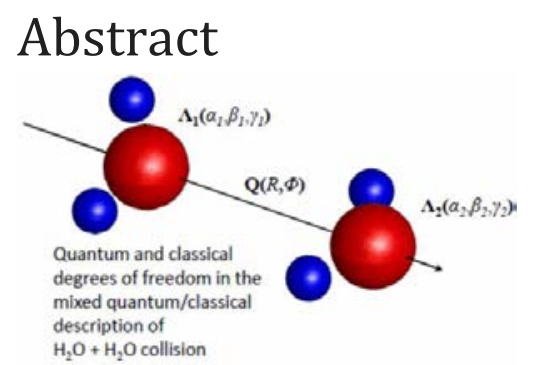

A mixed quantum/classical theory (MQCT) for the inelastic collision of two asymmetric-top rotor molecules is developed. In this method, the quantum state-to-state transitions between the rotational states of molecules (internal) are treated quantum mechanically using the time-dependent Schrodinger equation, whereas their relative translational motion (responsible for scattering) is treated classically, using the average trajectory approach. Two versions of the formula for transition matrix elements are presented: a straightforward approach 
that uses numerical multidimensional quadrature over all the internal degrees of freedom and a more standard analytic approach that uses the expansion of the PES over the basis set of spherical harmonics. Adaptation to the case of identical molecules scattering is presented and is applied to the rotational excitation of two water molecules, $\mathrm{H}_{2} \mathrm{O}+\mathrm{H}_{2} \mathrm{O}$, using the PES from recent literature. Calculations of collisional excitation from the ground state of the system into a number of low-lying excited rotational states are carried out in a broad range of energies. Analysis of computed opacity functions shows a rather unusual scattering regime, dominated by a strong anisotropic long-range interaction (dipole-dipole). The coupled-states (CS) approximation is tested and found to agree semiquantitatively (within a factor of 2) with the fully coupled version of the method. Differential cross sections for the elastic scattering indicate a very narrow forward scattering peak.

\section{Introduction}

The standard full-quantum theory of molecular inelastic scattering, known as coupled channel (CC) formalism, leads to a large system of coupled differential equations. $(1,2)$ The size of this system depends not only on the number of internal (rotational, vibrational) states of the molecules but also on the number of molecule-molecule orbital angular momentum states (partial waves) taken into account for the description of the scattering process. Numerically efficient methods and computer codes $(3,4)$ have been developed to solve this problem, which enabled computational studies of many important scattering processes. Computationally affordable cases include atom + diatomic,(5-7) diatomic + diatomic,(8-11) and atom + triatomic(12) collisions.

As molecules become larger and heavier, one has to deal with a higher density of the internal states (e.g., rotational levels in polyatomic molecules). The problem becomes severe at higher collision energies, when the number of accessible states becomes huge, simultaneously with a large number of partial waves required for the description of heavy-particle scattering. For example, full-quantum calculations for rotationally inelastic scattering of diatomic + triatomic systems are very demanding.(13-15) Likewise, inelastic scattering calculations for polyatomic molecules appear to be affordable only at small scattering energies.(16-19) As the focus of the community shifts toward more diverse and complex gas-phase chemistry,(20) and as electronic structure calculations of potential energy surfaces (PES) become affordable for larger molecules, one starts begging for the development of an alternative and more practical approach to molecular scattering.

In recent years, we developed a simplified mixed quantum/classical theory (MQCT) for inelastic scattering in which the relative motion of collision partners is treated approximately, classically, whereas their internal motion is still described rigorously using quantum mechanics. Since the scattering process is described by independent classical trajectories, there is no coupling between different values of orbital angular momentum, which reduces the sizes of matrices and systems of equations (now determined only by the number of internal states of the molecules). Further, speed up is achieved by intrinsic massive parallelism of the MQCT, where different trajectories are independent and can be propagated simultaneously using different processors, without any message passing. The resultant computational gain is very substantial, allowing inelastic scattering calculations for larger molecules and at higher collision energies, compared to the standard full-quantum approach.

We thoroughly tested this approach vs rigorous full-quantum calculations for an atom collided not only with diatomic $\left(\mathrm{CO}+\mathrm{He},(21) \mathrm{H}_{2}+\mathrm{He},(22)\right.$ and $\left.\mathrm{N}_{2}+\mathrm{Na}(23)\right)$, triatomic $\mathrm{H}_{2} \mathrm{O}+\mathrm{He},(24,25)$ tetratomic $\mathrm{CH}_{3}+\mathrm{He},(26)$ and polyatomic $\mathrm{HCOOCH}_{3}+\mathrm{He}(27)$ molecules but also for diatomic + diatomic collisions $\left(\mathrm{N}_{2}+\mathrm{H}_{2}(28)\right.$ and $\mathrm{H}_{2}+$ $\mathrm{H}_{2}(29)$ ). These systematic studies involved heavy and light collision partners, quenching and excitation at low and high collision energies, mostly rotational but also some vibrational transitions, including coherence effects for the elastic channel, scattering of identical partners, and computing both total and differential scattering cross sections. Typically, at intermediate and higher collision energies results of the MQCT are very close, often identical to the full-quantum results. But even at low collision energies, where classical approximation is not expected to be particularly accurate, the results of the MQCT are still reasonable (e.g., near excitation threshold). A proposal was made(26) to blend the full-quantum calculations at low collision energies, where they are indispensable and often affordable, with MQCT calculations at higher collision energies, where they are expected to be accurate, and where no other known method is practical. 
This paper is a capstone for our previous work, since here we expand the MQCT onto the most general case, a collision of two asymmetric-top rotor molecules, and use it for calculations of $\mathrm{H}_{2} \mathrm{O}+\mathrm{H}_{2} \mathrm{O}$ rotationally inelastic scattering.

The water molecule was a subject of several relevant studies using the full-quantum approach,(12-15) but those included either an $\mathrm{H}$ atom or an $\mathrm{H}_{2}$ molecule as its collision partner, which is much simpler. The $\mathrm{H}_{2} \mathrm{O}+\mathrm{H}_{2} \mathrm{O}$ system is so complicated that accurate scattering calculations have never been attempted for it, to our best knowledge. In fact, the two most popular scattering codes in use by the community today, MOLSCAT(3) and HIBRIDON,(4) do not even have capabilities of inelastic scattering calculations for the asymmetric-top + asymmetric-top systems. The only example of quantum $\mathrm{H}_{2} \mathrm{O}+\mathrm{H}_{2} \mathrm{O}$ scattering calculations is found in the early work of Clary,(30) but a very approximate infinite order sudden (IOS) assumption for collision was employed there. One other relevant example is a work of Buffa et al.(31) who also followed the spirit of the mixed quantum-classical approach but employed the impact-parameter approximation (basically, a straight-line assumption for the trajectory of motion), along with several other simplifications. (Namely, the identical particles collision symmetry was not incorporated, and no truly state-to-state cross sections were computed but rather thermally averaged cross sections for transitions in one molecule only, averaged over states of the other water molecule.)

Thus, this paper also breaks the grounds in the inelastic scattering calculations for the $\mathrm{H}_{2} \mathrm{O}+\mathrm{H}_{2} \mathrm{O}$ system, which is important on its own, for example, as a probe of conditions in cometary comas(32-34) and other astrophysical environments.(35)

\section{Theory}

\section{II-A Classical Degrees of Freedom}

In the MQCT the relative position of two scattering partners is given by vector $\mathbf{Q}$ that connects their centers of mass, as shown in Figure 1. Time evolution of this vector relative to the space-fixed reference frame (laboratory frame) describes the process of scattering. Spherical coordinates are used for this: $\mathbf{Q}=(R, \Theta, \Phi)$. It is possible to demonstrate $(22,23)$ that the average Ehrenfest potential, which governs this scattering process, possesses cylindrical symmetry for rotation around the vector $\mathbf{Q}$ which keeps the collision trajectory planar and permits to restrict consideration to one plane, e.g., the equatorial plane $\Theta=\pi / 2$ (horizontal plane in Figure 1), without loss of generality. The collision event can be thought of classically: At the initial moment of time two collision partners are in the asymptotic range, separated by a large distance $R=|\mathrm{Q}|$, which shortens during the time of collision and increases again as collision partners leave the interaction region. The deflection process is determined by the change of the azimuthal angle $\Phi$, which simply describes the rotation of $\mathbf{Q}$ in the equatorial plane, as collision partners approach each other, collide, and scatter. Thus, only two classical degrees of freedom are effectively used in this theory, $R$ and $\Phi$, together with their conjugate momenta $P_{R}$ and $P_{\Phi}$. Classical-like equations of motion for time evolution of these classical variables, $R(t), \Phi(t), P_{R}(t)$, and $P_{\Phi}(t)$, were derived and discussed in the recent literature. $(22,23,27,28)$ Here we also list them, for completeness, and in a slightly different form, found them to be more suitable for efficient numerical implementation:

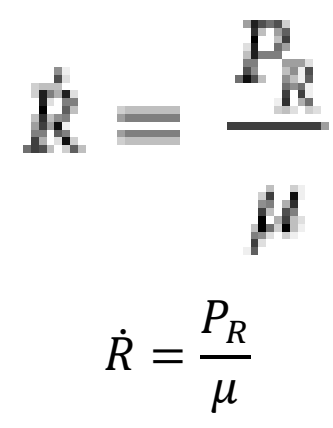




$$
\begin{aligned}
& +1+\frac{1}{H^{2}} \\
& \varphi=\frac{P_{\varphi}}{\mu \mathrm{R}^{2}}
\end{aligned}
$$

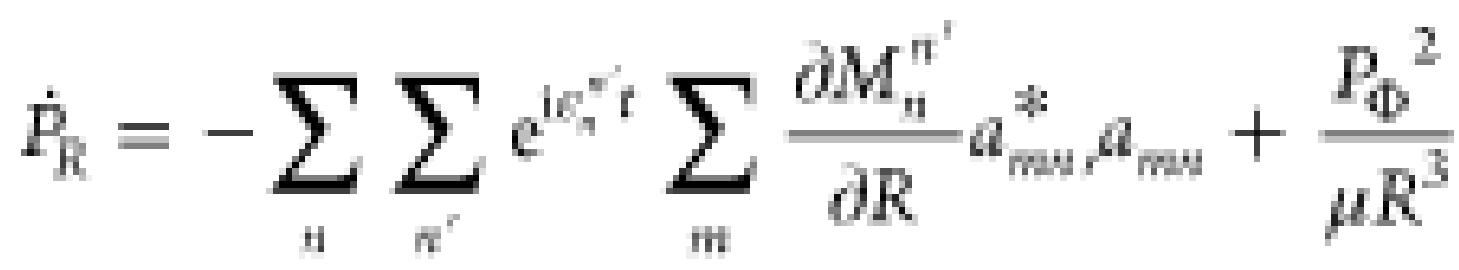

$$
\begin{aligned}
& \dot{P}_{R}=-\sum_{n} \sum_{n^{\prime}} e^{i \varepsilon_{n}^{n^{\prime}} t} \sum_{m} \frac{\partial M_{n}^{n^{\prime}}}{\vartheta R} a_{m n}^{*}, a_{m n}+\frac{P_{\Phi}^{2}}{\mu R^{3}} \\
& \dot{P}_{\Phi}=-\mathrm{i} \sum_{n} \sum_{\pi^{\prime}} \mathrm{e}^{\mathrm{i} e_{t}^{r^{\prime} t}} \sum_{m} M_{n}^{n^{\prime}}\left[a_{m-1, n^{\prime}}^{*} a_{m m} \sqrt{j^{\prime}\left(j^{\prime}+1\right)-m(m-1)}+a_{m+1, n^{\prime}}^{*} a_{m m} \sqrt{j^{\prime}\left(j^{\prime}+1\right)-m(m+1)}\right. \\
& \left.+a_{m m^{*}}^{*} a_{m-1, n} \sqrt{j(j+1)-m(m-1)}+a_{m m^{*}}^{*} a_{m+1, n} \sqrt{j(j+1)-m(m+1)}\right] / 2 \\
& \dot{P}_{\Phi}=-i \sum_{n} \sum_{n^{\prime}} e^{i \varepsilon_{n}^{n^{\prime}} t} \sum_{m} M_{n}^{n^{\prime}}\left[a_{m-1, n^{\prime}}^{*} a_{m n} \sqrt{j^{\prime}\left(j^{\prime}+1\right)-m(m-1)}\right. \\
& +a_{m+1, n^{\prime}}^{*} a_{m n} \sqrt{j^{\prime}\left(j^{\prime}+1\right)-m(m+1)} \\
& +a_{m n}^{*} a_{m-1, n^{\prime}} \sqrt{j^{\prime}\left(j^{\prime}+1\right)-m(m-1)} \\
& \left.+a_{m n}^{*} a_{m+1, n^{\prime}} \sqrt{j^{\prime}\left(j^{\prime}+1\right)-m(m+1)}\right] / 2
\end{aligned}
$$

In these equations, $\varepsilon_{n}^{n^{\prime}}=E_{n^{\prime}}-E_{n}$ is used to label energy differences between the initial (lower index) and the final (upper index) internal quantum states of the system, whereas $a_{m n}(t)$ represents time-evolving probability amplitudes for these states:

$$
\vec{a}_{m n}=-\mathrm{i} \sum_{n^{\prime}} \mathrm{e}^{i \sigma_{n}^{\prime \prime} t} M_{n^{\prime}}^{n} \cdot a_{m n^{\prime}}-\mathrm{i} \dot{\Phi}\left[a_{m-1, n} \sqrt{j(j+1)-m(m-1)}+a_{m+1, n} \sqrt{j(j+1)-m(m+1)}\right] / 2
$$




$$
\begin{aligned}
\dot{a}_{m n}=- & i \sum_{n^{\prime}} e^{i \varepsilon_{n^{\prime}}^{n} t} M_{n^{\prime}}^{n} a_{m n^{\prime}} \\
& -i \varphi\left[a_{m-1, n} \sqrt{j(j+1)-m(m-1)}\right. \\
& \left.+a_{m+1, n} \sqrt{j(j+1)-m(m+1)}\right] / 2
\end{aligned}
$$

The last term of eq 5 describes Coriolis coupling between states with $\Delta m= \pm 1$, driven by classical angular speed $\Phi(t)$. Neglecting this term leads to the coupled-states (CS) approximation within MQCT, while retaining this term corresponds to the fully coupled version of MQCT (or coupled-channel MQCT). Matrix $M_{n^{\prime \prime}}(R)$ in eqs $3-\underline{5}$ is a potential coupling matrix. Its $R$-dependent elements are real, are time independent, and are different for different values of $m$. The range of $m$ is between $-\min \left(j, j^{\prime}\right)$ and $+\min \left(j, j^{\prime}\right)$. The meaning of quantum numbers $m$, $j$, and a composite label $n$ are discussed next.

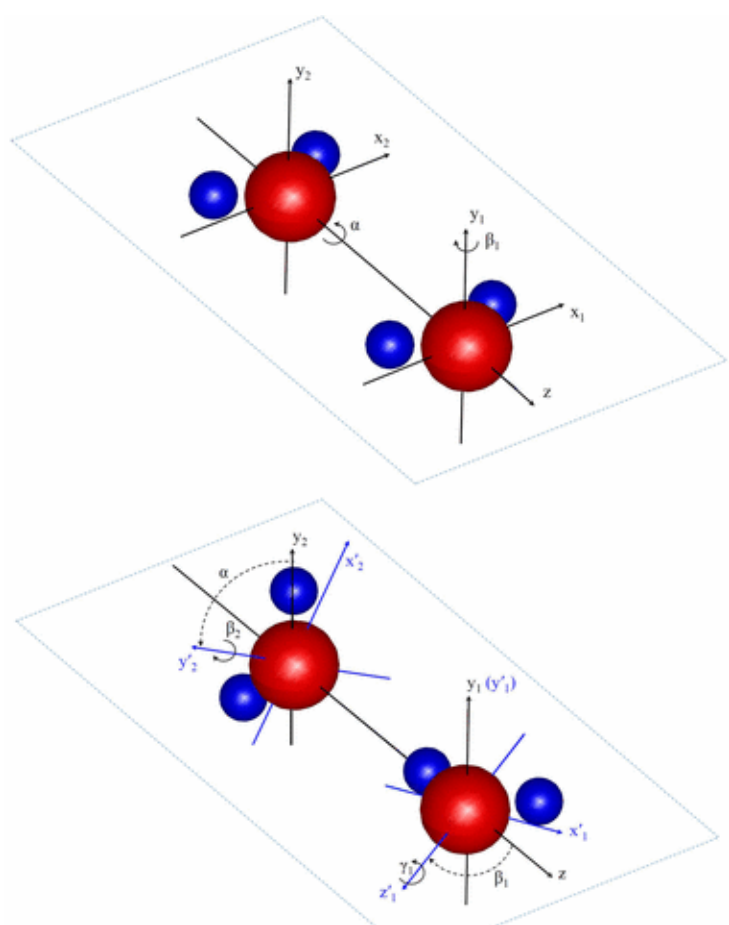

Figure 1. Euler angle rotations of two water molecules relative to the body-fixed frame tied to the instantaneous molecule-molecule vector $\mathbf{Q}$ treated classically. (a) Reference orientation with all angles set to zero. All axis labels are unprimed. The direction of first rotation is indicated for each molecule. (b) New orientations, after the first rotation of each molecule. New molecule-fixed axes, tied to the principal moments of inertia, are shown in blue and given primed labels. See text for further details.

\section{II-B Quantum Degrees of Freedom and the Reference Frame}

Here, for transparency, we will talk about two colliding water molecules (molecule one and molecule two), but since the water molecule is treated exactly as a general asymmetric top rotor, this theory is applicable to the collision of any two molecules. Adaptation of this theory to the scattering of two identical molecules is made further below.

So, rotation of each scattering partner is treated quantum mechanically and is described by a set of usual Euler angles: $\Lambda_{1}=\left(\alpha_{1}, \beta_{1}, \gamma_{1}\right)$ for molecule one and $\Lambda_{2}=\left(\alpha_{2}, \beta_{2}, \gamma_{2}\right)$ for molecule two, as shown in Figure 1 . Rotational states of each molecule are quantized, described by the corresponding wave functions: 


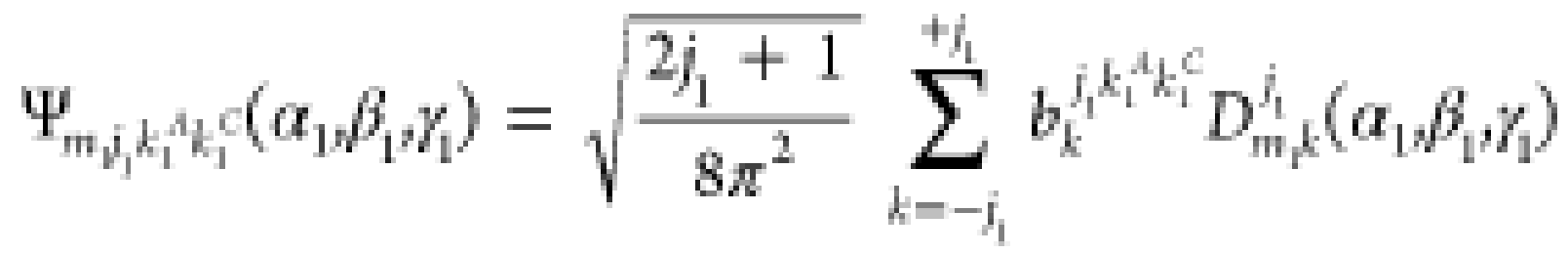

$$
\begin{aligned}
& \Psi_{m_{1} j_{1} k_{1}^{A} k_{1}^{C}}\left(\alpha_{1}, \beta_{1}, \gamma_{1}\right)=\sqrt{\frac{2 j_{1}+1}{8 \pi^{2}}} \sum_{k=-j_{1}}^{+j_{1}} b_{k}^{j_{1}{ }^{A_{1} k_{1}^{C}}} D_{m_{1} k}^{j_{1}}\left(\alpha_{1}, \beta_{1}, \gamma_{1}\right)
\end{aligned}
$$

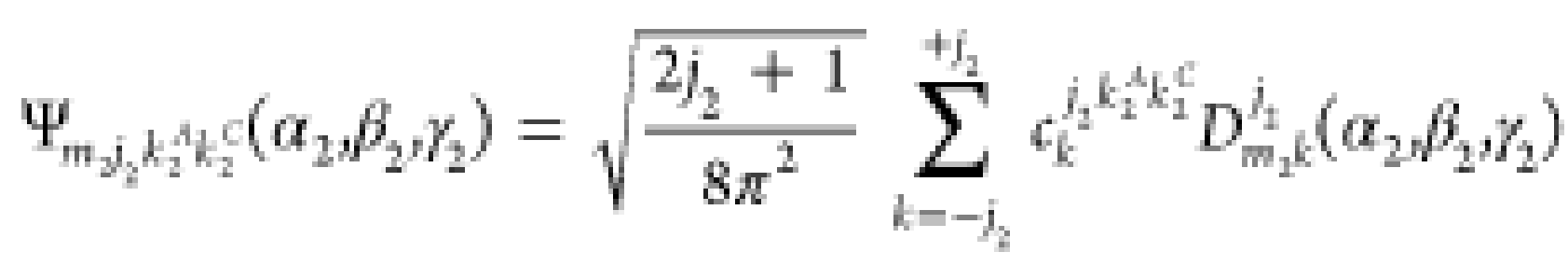

$$
\begin{aligned}
& \Psi_{m_{2} j_{2} k_{2}^{A} k_{2}^{C}}\left(\alpha_{2}, \beta_{2}, \gamma_{2}\right)=\sqrt{\frac{2 j_{2}+1}{8 \pi^{2}}} \sum_{k=-j_{2}}^{+j_{2}} b_{k}^{j_{2}{ }^{A_{2}^{k_{2}^{C}}}} D_{m_{2} j_{2}}^{j_{2}}\left(\alpha_{2}, \beta_{2}, \gamma_{2}\right)
\end{aligned}
$$

A set of expansion coefficients for each molecule, $b_{k} j_{1} k_{1}{ }^{A} k_{1} C$ and $c_{k^{j}} k_{2}{ }^{A} k_{2} C$, is obtained by diagonalization of an asymmetric-top Hamiltonian matrix in a corresponding basis set of Wigner $D$-functions, $D_{m 1} k_{1}\left(\alpha_{1}, \beta_{1}, \gamma_{1}\right)$ and $D_{m 2} k_{2}\left(\alpha_{2}, \beta_{2}, \gamma_{2}\right)$, respectively.(36) According to standard notation, rotational states of an asymmetric top are labeled (in addition to $j_{1}$ and $m_{1}$ for molecule one) by quantum numbers $k_{1}{ }^{A}$ and $k_{1} C$ that represent projections of $\mathbf{j}_{1}$ onto the principal axis of inertia with smallest and largest values of rotational constants, respectively (and similar for $k_{2}^{A}$ and $k_{2} C$ for the angular momentum $\mathbf{j}_{2}$ of molecule two, in addition to $j_{2}$ and $m_{2}$ ). Note that for water molecules this $A$-axis is also the axis of symmetry. It should be stressed that here we use the so-called body-fixed reference frame, where the $z$-axis is defined to pass through the centers of mass of two molecules (i.e., is tied to the classical molecule-molecule vector $\mathbf{Q}$ ). As collision progresses, this axis turns together with collision partners relative to the space-fixed reference frame (same as vector $\mathbf{Q}$ ), and this effect is incorporated into the equations of motion (eqs 1- $\underline{\text { ) }}$ as discussed in our earlier papers.(21,27) Projection $m_{1}$ of momentum $\mathbf{j}_{1}$ and projection $m_{2}$ of momentum $\mathbf{j}_{2}$ are made onto this body-fixed $z$-axis, or equivalently on $\mathbf{Q}$. The Euler angles $\Lambda_{1}=\left(\alpha_{1}, \beta_{1}, \gamma_{1}\right)$ and $\Lambda_{2}=$ $\left(\alpha_{2}, \beta_{2}, \gamma_{2}\right)$ are also defined relative to this body-fixed frame, using the so-called intrinsic rotations, or according to a $z-y^{\prime}-z^{\prime \prime}$ convention (note that these are different from extrinsic rotations discussed in Appendix A in the Supporting Information). Since the interaction between molecules is invariant under rotation around $z$-axes, one can set $\alpha_{1}=0$ and use only $\alpha_{2}=\alpha$. Figure 1 a gives the "reference" orientation of the system, when $\Lambda_{1}=(0,0,0)$ and $\Lambda_{2}=(0,0,0)$, and the symmetry axis of each molecule is aligned with the $z$-axis (or equivalently with $Q$ ). The direction for rotation of molecule two around the $z$-axis by angle $\alpha$ and the direction for rotation of molecule one around the $y_{1}$-axis by angle $\beta_{1}$ are also indicated in Figure $1 \mathrm{a}$. In Figure $1 \mathrm{~b}$, new positions of the molecule-fixed frames are shown, $\left(x_{1}{ }^{\prime}, z_{1}{ }^{\prime}\right)$ for molecule one and $\left(x_{2}{ }^{\prime}, y_{2}{ }^{\prime}\right)$ for molecule two, and the directions for the following rotations of each molecule are indicated: rotation around the $z_{1}^{\prime}$-axis by $\gamma_{1}$ (which is the last rotation for molecule one) and rotation around the $y_{2}^{\prime}$-axis by $\beta_{2}$ for molecule two. One remaining rotation of molecule two by angle $\gamma_{2}$ is not shown in Figure 1, since it occurs around the new axis $z_{2}^{\prime \prime}$.

The total angular momentum of two molecules $\mathbf{j}=\mathbf{j}_{1}+\mathbf{j}_{2}$ is also quantized in the MQCT. The corresponding eigenfunctions can be formally expressed through states of two coupled rotors: 


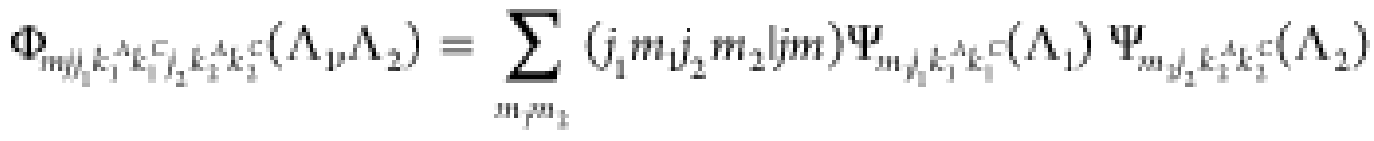

$$
\begin{aligned}
& \Phi_{m j j_{1} k_{1}^{A} k_{1}^{C} j_{2} k_{2}^{A} k_{2}^{C}(\Lambda 1, \Lambda 2)} \\
& =\sum_{m_{1} m_{2}}\left(j_{1} m_{1} j_{2} m_{2} \mid l j m\right) \psi m_{1} j_{1} k_{1}^{A} k_{1}^{C}\left(\Lambda_{1}\right) \psi m_{2} j_{2} k_{2}^{A} k_{2}^{C}\left(\Lambda_{2}\right)
\end{aligned}
$$

Coefficients of this expansion, $\left(j_{1} m_{1} j_{2} m_{2} \mid j m\right)$, the so-called Clebsch-Gordan coefficients,(37)are nonzero only if $m=m_{1}+m_{2}$ and $\left|j_{1}-j_{2}\right| \leq j \leq j_{1}+j_{2}$, where $m$ is the projection of $\mathbf{j}$ on $\mathbf{Q}$ so the sum in eq 8 should include all possible cases. A composite index $n$ is used to label the total set of quantum numbers for the system, $n=$ $\left\{j j k_{1}{ }^{A} k_{1} C j_{2} k_{2}{ }^{A} k_{2} C\right\}$. This is exactly the same index $n$ used in eqs $3-\underline{5}$; thus eq 8 gives the expression for $\Phi_{m n}$. It is also convenient to use $n_{1}=\left\{j_{1} k_{1}{ }^{A} k_{1} C\right\}$ and $n_{2}=\left\{j_{2} k_{2}{ }^{A} k_{2} C\right\}$ for the states of molecules one and two, respectively, so that $n=\left\{j n_{1} n_{2}\right\}$.

\section{II-C Potential Coupling Matrix Elements}

We have already demonstrated (and will confirm it one more time further below) that the potential coupling matrix $\mathbf{M}(R)$ is diagonal in $m$; i.e., its elements for transition $n m \leftarrow n^{\prime} m^{\prime}$ are nonzero only if $m=m^{\prime}$. However, the actual values of nonzero matrix elements depend on $m$. So, for given fixed $m$ consider the matrix element for transition $n \leftarrow n^{\prime}$ :

$$
\begin{aligned}
M_{n}^{H}(R)= & \left\langle\Phi_{m i n}\left(\Lambda_{1} \Lambda_{2}\right)\left|V\left(R_{j} \Lambda_{1} \Lambda_{2}\right)\right| \phi_{m i n}\left(\Lambda_{1} \Lambda_{2}\right)\right\rangle \\
& M_{n^{\prime}}^{n}(R)=\left\langle\varphi_{m n}\left(\Lambda_{1}, \Lambda_{2}\right) V\left(R, \Lambda_{1}, \Lambda_{2}\right) \varphi_{m n}\left(\Lambda_{1}, \Lambda_{2}\right)\right\rangle
\end{aligned}
$$

Here $V\left(R, \alpha_{1}, \beta_{1}, \gamma_{1}, \alpha_{2}, \beta_{2}, \gamma_{2}\right)$ is the potential energy hypersurface for the molecule-molecule interaction, expressed through the same variables: the molecule-molecule distance $R$ and two sets of Euler angles. From Figure 1 , we can see that without the loss of generality one of these angles can be eliminated, since the potential energy remains unchanged if the system, as a whole, rotates around $\mathbf{Q}$. So, $V\left(R, \alpha_{1}, \beta_{1}, \gamma_{1}, \alpha_{2}, \beta_{2}, \gamma_{2}\right)=V\left(R, 0, \beta_{1}, \gamma_{1}, \alpha, \beta_{2}, \gamma_{2}\right)$, where we defined the difference of angles as the new variable $\alpha=\alpha_{2}-\alpha_{1}$. In recent literature, a new PES for a water-water interaction was computed and presented using similar variables.(38,39) We adopted this surface for our calculations, as outlined in Appendix A. Other PESs for the water-water system of, perhaps, comparable accuracy are also available (e.g., Huang et al.(40))

One straightforward way of computing matrix elements is by the numerical five-dimensional quadrature (e.g., Gauss-Legendre method along each angular coordinate): 


$$
\begin{aligned}
& M_{n^{\prime}}^{\prime \prime}(R)= \int_{0}^{2 \pi} \mathrm{d} \alpha_{1} \int_{0}^{2 \pi} \mathrm{d} \alpha_{2} \int_{0}^{\pi} \sin \beta_{1} \mathrm{~d} \beta_{1} \int_{0}^{2 \pi} \mathrm{d} \gamma_{1} \int_{0}^{\pi} \sin \beta_{2} \mathrm{~d} \beta_{2} \int_{0}^{2 \pi} \mathrm{d} \gamma_{2} V\left(R, \Lambda_{v}, \Lambda_{2}\right) \Phi_{m n}^{*}\left(\Lambda_{1}, \Lambda_{2}\right) \\
& \Phi_{m n^{\prime}}\left(\Lambda_{1}, \Lambda_{2}\right) \\
&= 2 \pi \int_{-2 \pi}^{0} \mathrm{~d} \alpha \int_{0}^{\pi} \sin \beta_{1} \mathrm{~d} \beta_{1} \int_{0}^{2 \pi} \mathrm{d} \gamma_{1} \int_{0}^{\pi} \sin \beta_{2} \mathrm{~d} \beta_{2} \int_{0}^{2 \pi} \mathrm{d} \gamma_{2} V\left(R, 0, \beta_{1}, \gamma_{1}, \alpha, \beta_{2}, \gamma_{2}\right) \\
& \Phi_{m n}^{*}\left(0, \beta_{1}, \gamma_{1}, \alpha, \beta_{2}, \gamma_{2}\right) \Phi_{m n^{\prime}}\left(0, \beta_{1}, \gamma_{1}, \alpha, \beta_{2}, \gamma_{2}\right) \\
&= 2 \pi \int_{0}^{2 \pi} \mathrm{d} \alpha \int_{0}^{\pi} \sin \beta_{1} \mathrm{~d} \beta_{1} \int_{0}^{2 \pi} \mathrm{d} \gamma_{1} \int_{0}^{\pi} \sin \beta_{2} \mathrm{~d} \beta_{2} \int_{0}^{2 \pi} \mathrm{d} \gamma_{2} V\left(R, 0, \beta_{1}, \gamma_{1}, \alpha, \beta_{2}, \gamma_{2}\right) \\
& \Phi_{m m}^{*}\left(0, \beta_{1}, \gamma_{1}, \alpha, \beta_{2}, \gamma_{2}\right) \Phi_{m n^{\prime}}\left(0, \beta_{1}, \gamma_{1}, \alpha, \beta_{2}, \gamma_{2}\right) \\
& M_{n^{\prime}}^{n}(R)= \int_{0}^{2 \pi} \mathrm{d} \alpha_{2} \int_{0}^{\pi} \sin \beta_{1} \mathrm{~d} \beta_{1} \int_{0}^{2 \pi} \mathrm{d} \gamma_{1} \int_{0}^{\pi} \sin \beta_{2} \mathrm{~d} \beta_{2} \int_{0}^{2 \pi} \mathrm{d} \gamma_{1} V\left(R, \Lambda_{1}, \Lambda_{2}\right) \Phi_{m n}\left(\Lambda_{1}, \Lambda_{2}\right) \Phi_{m n \prime}\left(\Lambda_{1}, \Lambda_{2}\right) \\
& \quad=2 \pi \int_{-2 \pi}^{0} \mathrm{~d} \alpha \int_{0}^{\pi} \sin \beta_{1} \mathrm{~d} \beta_{1} \int_{0}^{2 \pi} \mathrm{d} \gamma_{1} \int_{0}^{\pi} \sin \beta_{2} \mathrm{~d} \beta_{2} \int_{0}^{2 \pi} \mathrm{d} \gamma_{2} V\left(R, 0, \beta_{1}, \gamma_{1}, \alpha, \beta_{2}, \gamma_{2}\right) \Phi_{m n}^{*}\left(0, \beta_{1}, \gamma_{1}, \alpha, \beta_{2}, \gamma_{2}\right) \Phi_{m n \prime}\left(0, \beta_{1}, \gamma_{1}, \alpha, \beta_{2}, \gamma_{2}\right) \\
& \quad=2 \pi \int_{0}^{2 \pi} \mathrm{d} \alpha \int_{0}^{\pi} \sin \beta_{1} \mathrm{~d} \beta_{1} \int_{0}^{2 \pi} \mathrm{d} \gamma_{1} \int_{0}^{\pi} \sin \beta_{2} \mathrm{~d} \beta_{2} \int_{0}^{2 \pi} d \gamma_{2} V\left(R, 0, \beta_{1}, \gamma_{1}, \alpha, \beta_{2}, \gamma_{2}\right) \Phi_{m n}^{*}\left(0, \beta_{1}, \gamma_{1}, \alpha, \beta_{2}, \gamma_{2}\right) \Phi_{m n^{\prime}}\left(0, \beta_{1}, \gamma_{1}, \alpha, \beta_{2}, \gamma_{2}\right)
\end{aligned}
$$


We not only implemented this method in our calculations but also followed an alternative approach described below, since that second method could be more efficient computationally and, possibly, would allow more straightforward comparison with standard full-quantum calculations (if they would appear in the future).

Namely, in a method used normally in the typical full-quantum scattering calculations, the multidimensional potential is expanded over a basis set of suitable functions. Following Szalewicz, $(38,39)$ we used

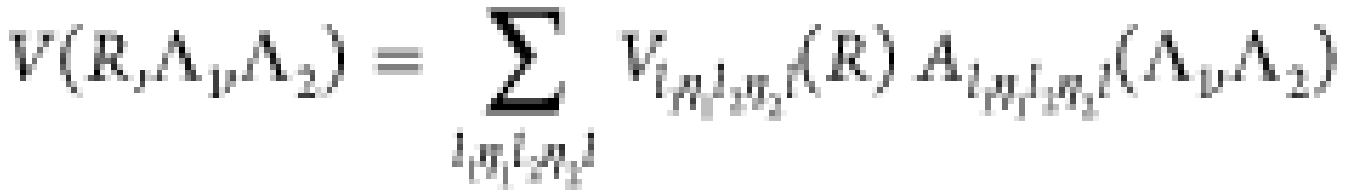

$$
\begin{aligned}
& V\left(R, \Lambda_{1}, \Lambda_{2}\right)=\sum_{l_{1} \eta_{1} l_{2} \eta 2 l} V_{l_{1} \eta_{1} l_{2} \eta l}(R) A_{l_{1} \eta_{1} l_{2} \eta_{2} l}\left(\Lambda_{1}, \Lambda_{2}\right)
\end{aligned}
$$

where the real functions are employed, as follows:

$$
\begin{gathered}
A_{l_{1} \eta_{1} l_{l} \eta_{l}}\left(\Lambda_{1} \Lambda_{2}\right)=\sum_{m_{l}} \frac{\sqrt{\left(2 l_{1}+1\right)\left(2 l_{2}+1\right)}}{8 \pi^{2}}\left(l_{1} m_{l} l_{2}-m_{l} l 0\right) D_{m_{l} \eta_{1}}^{l_{1}}\left(\Lambda_{1}\right) D_{-m_{l} \eta_{1}}^{l_{1}}\left(\Lambda_{2}\right) \\
A_{l_{1} \eta_{1} l_{2} \eta_{2} l}\left(\Lambda_{1}, \Lambda_{2}\right) \\
=\sum_{m_{l}} \frac{\sqrt{\left(2 l_{1}+1\right)\left(2 l_{2}+1\right)}}{8 \pi^{2}}\left(l_{1} m_{l} l_{2}\right. \\
\left.-m_{l} l 0\right) D_{m_{l} \eta_{1}}^{l_{1}}\left(\Lambda_{1}\right) D_{-m_{l} \eta_{2}}^{l_{2}}\left(\Lambda_{2}\right)
\end{gathered}
$$

Here the labels $l_{1}, I_{2}$ and $\eta_{1}, \eta_{2}$ are analogues of the quantum numbers $j$ and $k$ of two molecules, respectively. The range of $m_{l}$ is between $-\min \left(l_{1}, l_{2}\right)$ and $+\min \left(l_{1}, l_{2}\right)$. Note that to use this method, one should still compute the multidimensional integrals to determine the values of expansion coefficients $V_{l} \eta_{1} l_{2} \eta_{2} I(R)$ by projecting the PES $V\left(R, \Lambda_{1}, \Lambda_{2}\right)$ onto the "basis functions" $A_{h} \eta_{1} l_{2} \eta_{2} l\left(\Lambda_{1}, \Lambda_{2}\right)$. But if the expansion coefficients are determined on a grid of points along $R$, then those can be splined and the values of all matrix elements are computed quickly at any point along $R$ (using the formula derived below). This is different from the first method, eq 10 , where each matrix element should be precomputed on the $R$-grid and then splined between those points. Depending on complexity of the PES, and the number of states of the system, the first or the 
second method can be better (faster). In order to test our theory and new code, we implemented both methods and made sure they gave identical results.

In order to use the expansion of eq 11 in eq 9, we have to be able to compute the following matrix elements:

$$
\begin{array}{r}
\left\langle\Phi_{m !}\left(\Lambda_{1} \Lambda_{2}\right)\left|A_{l_{1} h_{1} h_{1}}\left(\Lambda_{p} \Lambda_{2}\right)\right| \Phi_{m n^{\prime}}\left(\Lambda_{1} \Lambda_{2}\right)\right\rangle \\
\left\langle\Phi_{m n}\left(\Lambda_{1}, \Lambda_{2}\right)\left|A_{l_{1} \eta_{1} l_{2} \eta_{l}}\left(\Lambda_{1}, \Lambda_{2}\right)\right| \Phi_{m n}\left(\Lambda_{1}, \Lambda_{2}\right)\right\rangle
\end{array}
$$

by expressing them analytically through the coefficients $b_{k 1} j_{1} k_{1}{ }^{A} k_{1}{ }^{C}$ and $c_{k} j_{2} k_{2}{ }^{A} k_{2} C_{\text {of }}$ eqs 6 and $\underline{7}$. The derivations are relatively straightforward but somewhat lengthy and, for the sake of transparency, are presented separately in Appendix B. The formula used to calculate the matrix element's $m_{{ }^{\prime}}{ }^{\prime \prime}(R)$ equation through the expansion coefficients $V_{l} \eta_{1} l_{2} \eta_{2} I(R)$ is also derived in Appendix B.

\section{II-D Identical Particle Exchange Symmetry}

In addition to the wave function $\Phi_{m n}\left(\Lambda_{1}, \Lambda_{2}\right)$ defined by eq 8 , consider another wave function $\tilde{\Phi}_{m n}\left(\Lambda_{1}, \Lambda_{2}\right)$ obtained from it by swapping the molecules one and two. Several arguments should be taken into account. First of all, since the identity of molecules is retained, this swap, obviously, inverts the direction of the classical vector $\mathbf{Q}$ in space, namely, $(X, Y, Z) \rightarrow(-X,-Y,-Z)$. Second, for an arbitrary orientation of molecule in space defined by some $\Lambda=(\alpha, \beta, \gamma)$, the inversion of $\mathbf{Q}$ changes the values of coordinates to new values:(35) $\tilde{\Lambda}=(\pi+\alpha, \pi-\beta, \pi-\gamma)$, and this concerns each molecule. Third, if we swap two molecules, each takes the quantum state of the partner and its corresponding wave function. The expansion coefficients used to give wave functions of the entire system in eq 8, Clebsch-Gordan coefficients, should be modified accordingly. All these features are incorporated into the new total wave function of the system, as follows:

$$
\begin{aligned}
& \Phi_{m}\left(\Lambda_{1} \Lambda_{2}\right)=\Phi_{m}\left(\Lambda_{2} \bar{A}_{1}\right)
\end{aligned}
$$

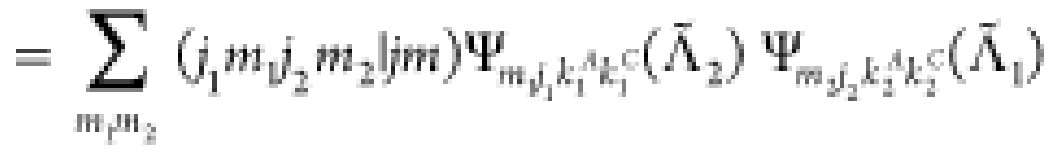

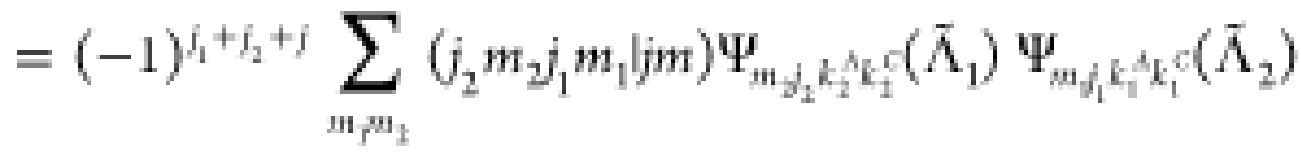




$$
\begin{aligned}
& \Phi_{m n}\left(\Lambda_{1}, \Lambda_{2}\right)=\Phi_{m n}\left(\widetilde{\Lambda}_{2}, \tilde{\Lambda}_{1}\right) \\
& =\sum_{m_{1} m_{2}}\left(j_{1} m_{1} j_{2} m_{2} \mid j m\right) \Psi_{m_{1} j_{1} k_{1}^{A} k_{1}^{C}}\left(\tilde{\Lambda}_{2}\right) \Psi_{m_{2} j_{2} k_{2}^{A} k_{2}^{C}}\left(\tilde{\Lambda}_{1}\right) \\
& =(-1)^{j_{1}+j_{2}+j} \sum_{m_{1} m_{2}}\left(j_{2} m_{2} j_{1} m_{1} \mid j m\right) \Psi_{m_{2} j_{2} k_{2}^{A} k_{2}^{C}}\left(\widetilde{\Lambda}_{1}\right) \Psi_{m_{1} j_{1} k_{1}^{A} k_{1}^{C}}\left(\tilde{\Lambda}_{2}\right)
\end{aligned}
$$

The goal here is to make this expression look as the original formula of eq 8 multiplied by a phase factor (or, actually, to derive the expression for this phase factor). In the second part of eq 14, we have already inverted the order of states in the Clebsch-Gordan coefficient,(36)which gave a part of this factor, $(-1) j_{1}+j_{2}+j$ The remaining task is to transform individual asymmetric-top wave functions of each molecule. Using their definitions, eqs 6 and $\underline{7}$, we can write (e.g., for the molecule two, now in the original rotational state of the molecule one):

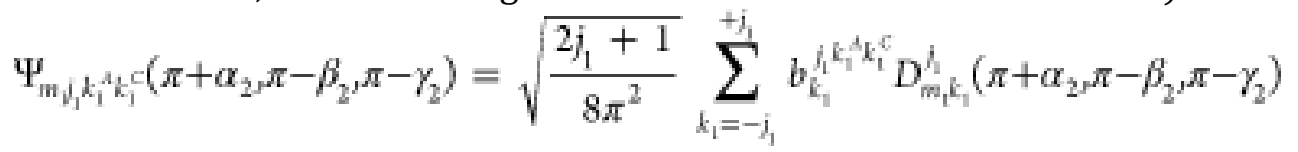

$$
\begin{aligned}
& =\sqrt{\frac{2 j_{1}+1}{8 \pi^{2}}} \sum_{k_{1}=-j_{1}}^{+j_{1}} b_{k_{1}}^{j_{1} k_{1}{ }_{1} k_{1} k_{1}^{c}}(-1)^{j_{1}+k_{1}} D_{m_{1},-k_{1}}^{j_{1}}\left(\alpha_{2}, \beta_{2}, \gamma_{2}\right) \\
& =(-1)^{i_{1}} \sqrt{\frac{2 j_{1}+1}{8 \pi^{2}}} \sum_{k_{1}=-j_{1}}^{+j_{1}}(-1)^{k_{1}} b_{k_{1}}^{j_{1}, k_{1}^{\lambda_{1}} k_{1}^{c}} D_{m_{1},-k_{1}}^{j_{1}}\left(\alpha_{2}, \beta_{2}, \gamma_{2}\right)
\end{aligned}
$$

$$
\begin{aligned}
& \Psi m_{1} j_{1} k_{1}^{A} k_{1}^{C}\left(\pi+\alpha_{2}, \pi-\beta_{2}, \pi-\gamma_{2}\right) \\
& \begin{array}{l}
=\sqrt{\frac{2 j_{1}+1}{8 \pi^{2}} \sum_{k_{1}=-j_{1}}^{+j_{1}} b_{k_{1}}^{j_{1} k_{1}^{A} k_{1}^{C}} D_{m_{1} k_{1}}^{j_{1}}\left(\pi+\alpha_{2}, \pi-\beta_{2}, \pi-\gamma_{2}\right)} \\
=\sqrt{\frac{2 j_{1}+1}{8 \pi^{2}} \sum_{k_{1}=-j_{1}}^{+j_{1}} b_{k_{1}}^{j_{1} k_{1}^{A} k_{1}^{C}}(-1)^{j_{1} k_{1}} D_{m_{1}-k_{1}}^{j_{1}}\left(\alpha_{2}, \beta_{2}, \gamma_{2}\right)} \\
=(-1)^{j_{1}} \sqrt{\frac{2 j_{1}+1}{8 \pi^{2}}} \sum_{k_{1}=-j_{1}}^{+j_{1}}(-1)^{k_{1}} b_{k_{1}}^{j_{1} k_{1}^{A} k_{1}^{C}} D_{m_{1}-k_{1}}^{j_{1}}\left(\alpha_{2}, \beta_{2}, \gamma_{2}\right)
\end{array}
\end{aligned}
$$

Here we not only used a property of the Wigner $D$-function,(36) which gave the factor of $(-1)^{j_{1}}+k_{1}$ but also swapped $D$-functions with positive and negative values of $\Lambda=(\alpha, \beta, \gamma)$. The same 
considerations were applied to the wave function of molecule one (in the original state of molecule two), which gave the factor $(-1) j_{2}+k_{2}$.

Note that a product of $(-1) j_{1}$ and $(-1) j_{2}$ obtained in eq 15 multiplied by the factor

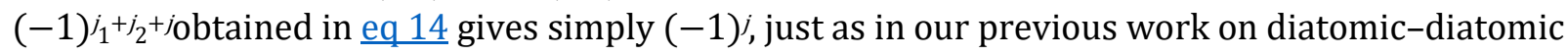
scattering.(29) The factors $(-1)^{k_{1}}$ and $(-1)^{k_{2}}$ disappear if we split the total sum over $k$ into two, one of which includes only the terms with even values of $k$ (both positive and negative) including zero, while the other includes only the terms with odd values of $k$, namely, from eq 15:

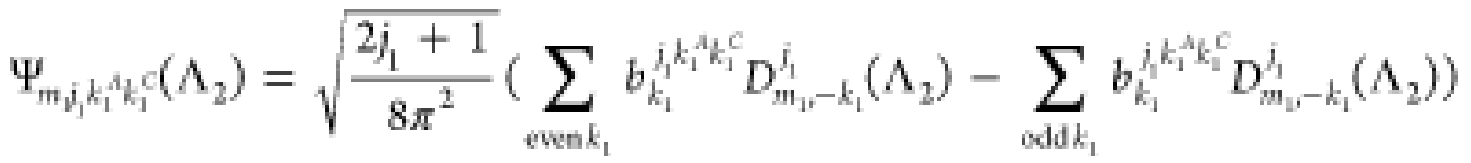

$$
\begin{aligned}
& \Psi_{m_{1} j_{1} k_{1}^{A} k_{1}^{C}}\left(\Lambda_{2}\right) \\
& =\sqrt{\frac{2 j_{1}+1}{8 \pi^{2}}}\left(\sum_{\text {even } k_{1}} b_{k_{1}}^{j_{1} k_{1}^{A} k_{1}^{C}} D_{m_{1}-k_{1}}^{j_{1}}\left(\Lambda_{2}\right)\right. \\
& -\sum_{\text {odd } k_{1}} b_{k_{1}}^{j_{1} k_{1}^{A} k_{1}^{C}} D_{m_{1}-k_{1}}^{j_{1}}\left(\Lambda_{2}\right)
\end{aligned}
$$

The overall range of $k_{1}$-values is still $-j_{1} \leq k_{1} \leq+j_{1}$ and is similar for the other molecule.

Importantly, as it is shown in Appendix C, no rotational states of the asymmetric-top rotor include both even and odd $k_{1}$-values in the expansion (i.e., even and odd $k_{1}$-values do not mix). Some states, called para-states, are described by the first sum in eq 16, while other states, called ortho-states, are described by the second sum in eq 16 , which permits us to simplify it as follows:

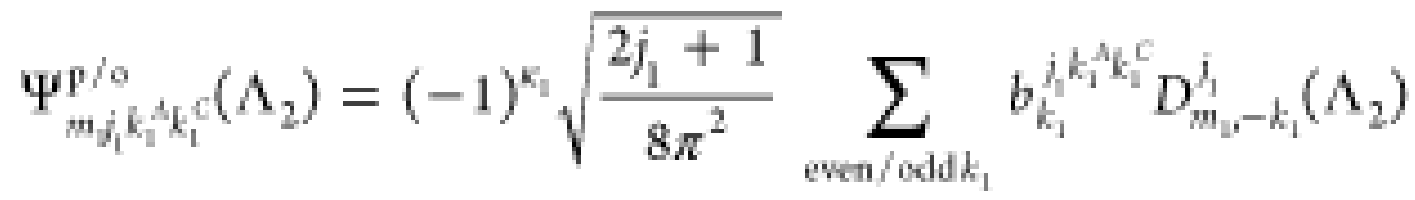

$$
\begin{aligned}
& \Psi_{m_{1} j_{1} k_{1}^{A} k_{1}^{C}}^{p / o}\left(\Lambda_{2}\right)=(-1)^{\kappa_{1}} \sqrt{\frac{2 j_{1}+1}{8 \pi^{2}}} \sum_{\text {even } / \text { odd } k_{1}} b_{k_{1}}^{j_{1} k_{1}^{A} k_{1}^{C}} D_{m_{1}-k_{1}}^{j_{1}}\left(\Lambda_{2}\right)
\end{aligned}
$$

The value of $\kappa_{1}=0$ is taken simultaneously with even $k_{1}$-values for para-states, while $\kappa_{1}=1$ is taken with odd $k_{1}$-values for ortho-states and is similar for the other molecule.

Moreover, as it is also shown in Appendix $\mathrm{C}$, the coefficients of expansion obey the following property: $b_{k 1} j_{1} k_{1}{ }^{A} k_{1} C=p_{1} b_{-k 1} j_{1} k_{1}{ }^{A} k_{1} C$ (for all values of $k_{1}$ in the range $-j_{1} \leq k_{1} \leq+j_{1}$ ), where $p_{1}= \pm 1$ 
determines the inversion parity of the rotational states of the molecule. This is equivalent to paring $D$-functions with different signs of $k_{1}$ into new basis functions of positive and negative parity:

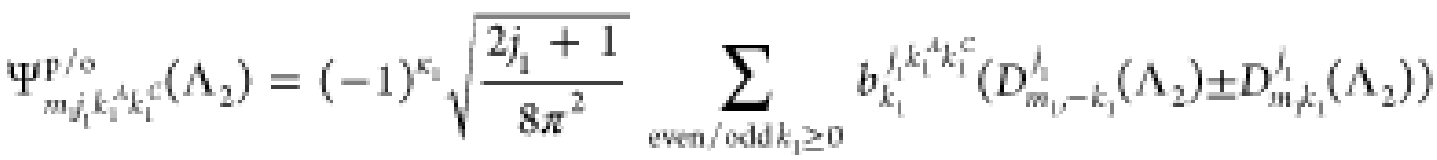

$$
\begin{aligned}
& =(-1)^{k_{1}} p_{1} \sqrt{\frac{2 j_{1}+1}{8 \pi^{2}}} \sum_{\text {evere } / \text { odd } k_{1} \geq 0} b_{k_{1}}^{j_{1} k_{1}^{\lambda_{1} k_{1}^{c}}}\left(D_{m_{1} k_{1}}^{j_{1}}\left(\Lambda_{2}\right) \pm D_{m_{1},-k_{1}}^{j_{1}}\left(\Lambda_{2}\right)\right) \\
& =(-1)^{K_{1}} p_{1} \Psi_{m_{j, j} k_{1}^{A_{k}} c}^{ \pm p / o}\left(\Lambda_{2}\right) \\
& \Psi_{m_{1} j_{1} k_{1}^{A} k_{1}^{C}}^{p}\left(\Lambda_{2}\right)
\end{aligned}
$$

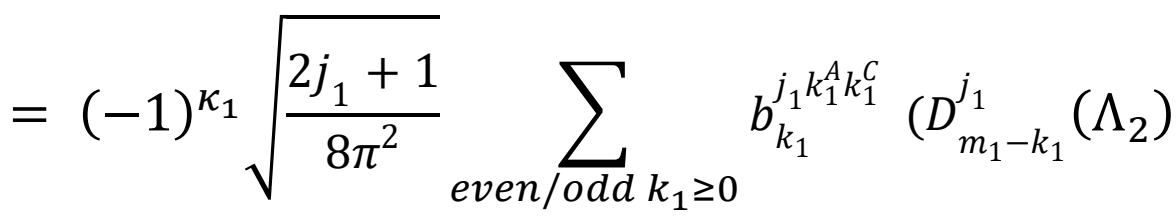

$$
\begin{aligned}
& \left. \pm D_{m_{1}, k_{1}}^{j_{1}}\left(\Lambda_{2}\right)\right) \\
& =(-1)^{\kappa_{1}} P_{1} \sqrt{\frac{2 j_{1}+1}{8 \pi^{2}}} \sum_{\text {even } / \text { odd } k_{1} \geq 0} b_{k_{1}}^{j_{1} k_{1}^{A} k_{1}^{C}}\left(D_{m_{1}-1}^{j_{1}}\left(\Lambda_{2}\right)\right. \\
& \left.\left. \pm D_{m_{1}, k_{1}}^{j_{1}}\left(\Lambda_{2}\right)\right)=(-1)^{\kappa_{1}} p_{1} \Psi_{m_{1} j_{1} k_{1}^{A} k_{1}^{C}}^{p / \Lambda_{2}}\right)
\end{aligned}
$$

Note that here the range of $k_{1}$-values is reduced to non-negative values only, just $0 \leq k_{1} \leq j_{1}$, and is similar for the other molecule.

Overall, we can write for the wave function of two swapped molecules:

$$
\begin{aligned}
& \phi_{\min }\left(\Lambda_{0} \Lambda_{2}\right)
\end{aligned}
$$

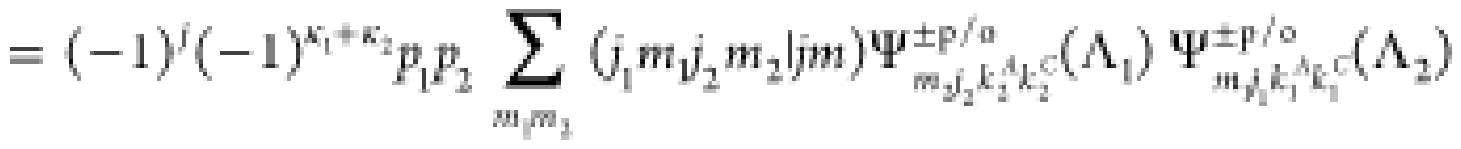

$$
\begin{aligned}
& \Phi_{m n}\left(\Lambda_{1}, \Lambda_{2}\right)=(-1)^{j}(-1)^{\kappa_{1}+\kappa_{2}} p_{1} p_{2}
\end{aligned}
$$




$$
\sum_{m_{1} m_{2}}\left(j_{1} m_{1} j_{2} m_{2} \mid j m\right) \Psi_{m_{2} j_{2} k_{2}^{A} k_{2}^{C}}^{ \pm p / o}\left(\Lambda_{1}\right) \Psi_{m_{1} j_{1} k_{1}^{A} k_{1}^{C}}^{ \pm p / o}\left(\Lambda_{2}\right)
$$

Note that in eqs 18 and 19 , we introduced new notation to label the molecular eigenstates of the given parity, $\Psi \pm(\Lambda)$. These can also be used for the original wave functions (before the swap), since the property $b_{k 1} j_{1} k_{1}^{A}{ }_{k_{1}} C=p_{1} b_{-k 1} j_{1} k_{1}{ }^{A} k_{1} C$ is valid in either case:

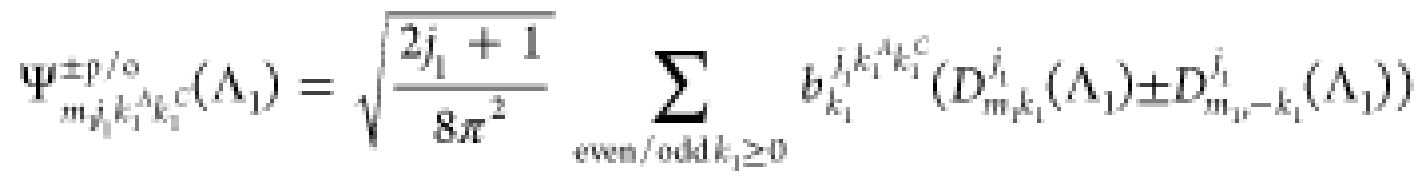

$$
\begin{aligned}
& \Psi_{m_{1} j_{1} k_{1}^{A} k_{1}^{C}}^{ \pm p / o}\left(\Lambda_{1}\right) \\
& =\sqrt{\frac{2 j_{1}+1}{8 \pi^{2}}} \sum_{\text {even } / \text { odd } k_{1} \geq 0} b_{k_{1}}^{j_{1} k_{1}^{A} k_{1}^{C}}\left(D_{m_{1} k_{1}}^{j_{1}}\left(\Lambda_{1}\right) \pm D_{m_{1},-k_{1}}^{j_{1}}\left(\Lambda_{1}\right)\right)
\end{aligned}
$$

A similar expression is shown for molecule two. It should be stressed that the para-/ortho-states (with respect to the values of $k$ in the basis) and the states of two parities (with respect to inversion) occur in any asymmetric-top molecule, not just in water, and irrespective of the process of collision with any given partner (e.g., in the absence of any collision partner).

Finally, the symmetrized overall wave function of the system of two molecules can be written as positive or negative superposition of the original and swapped wave functions:

$$
\begin{aligned}
& \Phi_{m=1}^{ \pm}\left(\Lambda_{p} \Lambda_{2}\right)=\frac{\Phi_{m}\left(\Lambda_{1} \Lambda_{2}\right) \pm \Phi_{m n}\left(\Lambda_{v} \Lambda_{2}\right)}{\sqrt{2\left(1+\delta_{12}\right)}} \\
& =\frac{\Phi_{\min }\left(\Lambda_{1} \Lambda_{2}\right) \pm(-1)(-1)^{k_{1}+\kappa_{1}} p_{1} p_{2} \Phi_{m i}\left(\Lambda_{1} \Lambda_{2}\right)}{\sqrt{2\left(1+\delta_{12}\right)}} \\
& =\frac{1}{\sqrt{2\left(1+\delta_{12}\right)}}\left(\Phi_{\min }\left(\Lambda_{p} \Lambda_{2}\right) \pm p \Phi_{m i n}\left(\Lambda_{p} \Lambda_{2}\right)\right)
\end{aligned}
$$




$$
\begin{aligned}
\Phi_{m n}^{ \pm}\left(\Lambda_{1}, \Lambda_{2}\right)=\frac{\Phi_{m n}\left(\Lambda_{1}, \Lambda_{2}\right) \pm \widetilde{\Phi}_{m n}\left(\Lambda_{1}, \Lambda_{2}\right)}{\sqrt{2\left(1+\delta_{12}\right)}} \\
=\frac{\Phi_{m n}\left(\Lambda_{1}, \Lambda_{2}\right) \pm(-1)^{j}(-1) \kappa_{1}+\kappa_{2} p_{1} p_{2} \Phi_{m \tilde{n}}\left(\Lambda_{1}, \Lambda_{2}\right)}{\sqrt{2\left(1+\delta_{12}\right)}} \\
=\frac{1}{\sqrt{2\left(1+\delta_{12}\right)}}\left(\Phi_{m n}\left(\Lambda_{1}, \Lambda_{2}\right) \pm p \Phi_{m \tilde{n}}\left(\Lambda_{1}, \Lambda_{2}\right)\right)
\end{aligned}
$$

Here $\tilde{n}=\left\{j n_{2} n_{1}\right\}$, where particles one and two have been swapped. We also introduced the total inversion parity of the overall wave function of two molecules as $p= \pm(-1)^{j}(-1)^{\kappa_{1}}+\kappa_{2} p_{1} p_{2}$. The normalization coefficient includes the Kronecker symbol for the states of two molecules: $\delta_{12}=$ $\delta_{j 1} k_{1}{ }^{A} k_{1}{ }^{C}{ }_{, j 2} k_{2}{ }^{A} k_{2} C$. One can see that many combinations of the para- and ortho-states of both parities of the two molecules are possible. The next section discusses which state-to-state transitions are allowed/forbidden and what are the corresponding matrix elements.

\section{II-E Transitions in the Case of Identical Particles}

The exchange parity of the overall wave function of the two-molecule system is very handy because it appears that state-to-state transitions $n \rightarrow n^{\prime}$ are allowed only between states of the same parity, which restricts the values of the final quantum numbers $n=\left\{j n_{1} n_{2}\right\}$ for a chosen initial state $n^{\prime}=$ $\left\{f_{1}^{\prime} n_{2}^{\prime}\right\}$, for every value of $m$. Let us demonstrate this. Consider the matrix element where exchange parities are the same (both are positive, or both are negative):

$$
\begin{aligned}
& M_{n^{\prime}}^{n( \pm)}(R) \\
& =\left\langle\Phi_{m v}^{ \pm}\left(\Lambda_{1}, \Lambda_{2}\right)\left|V\left(R, \Lambda_{v} \Lambda_{2}\right)\right| \Phi_{m v}^{ \pm}\left(\Lambda_{1}, \Lambda_{2}\right)\right\rangle \\
& =\left\langle\frac{\Phi_{m n}\left(\Lambda_{1}, \Lambda_{2}\right) \pm \Phi_{m n}\left(\Lambda_{1}, \Lambda_{2}\right)}{\sqrt{2\left(1+\delta_{12}\right)}}\left|V\left(R, \Lambda_{1}, \Lambda_{2}\right)\right| \frac{\Phi_{m n^{\prime}}\left(\Lambda_{1}, \Lambda_{2}\right) \pm \Phi_{m n^{\prime}}\left(\Lambda_{1}, \Lambda_{2}\right)}{\sqrt{2\left(1+\delta_{12}^{\prime}\right)}}\right\rangle \\
& =\frac{1}{2 \sqrt{\left(1+\delta_{12}^{\prime}\right)\left(1+\delta_{12}\right)}}\left(\left\langle\Phi_{m m}\left(\Lambda_{1}, \Lambda_{2}\right)\left|V\left(R, \Lambda_{1}, \Lambda_{2}\right)\right| \Phi_{m n^{\prime}} \cdot\left(\Lambda_{1}, \Lambda_{2}\right)\right\rangle\right. \\
& +\left\langle\tilde{\Phi}_{m m}\left(\Lambda_{1}, \Lambda_{2}\right)\left|V\left(R, \Lambda_{1}, \Lambda_{2}\right)\right| \tilde{\Phi}_{m n} \cdot\left(\Lambda_{1}, \Lambda_{2}\right)\right\rangle \\
& \pm\left\langle\Phi_{m n}\left(\Lambda_{v}, \Lambda_{2}\right)\left|V\left(R, \Lambda_{1}, \Lambda_{2}\right)\right| \tilde{\Phi}_{m \pi} \cdot\left(\Lambda_{1}, \Lambda_{2}\right)\right\rangle \\
& \left. \pm\left\langle\tilde{\Phi}_{m n}\left(\Lambda_{1}, \Lambda_{2}\right)\left|V\left(R, \Lambda_{1}, \Lambda_{2}\right)\right| \Phi_{m r}\left(\Lambda_{1}, \Lambda_{2}\right)\right\rangle\right)
\end{aligned}
$$




$$
\begin{aligned}
& M_{n}^{n( \pm)}(R)=\left\langle\Phi_{m n}^{ \pm}\left(\Lambda_{1}, \Lambda_{2}\right)\left|V\left(R, \Lambda_{1}, \Lambda_{2}\right)\right| \Phi_{m n^{\prime}}^{ \pm}\left(\Lambda_{1}, \Lambda_{2}\right)\right\rangle \\
& =\left\langle\frac{\Phi_{m n}\left(\Lambda_{1}, \Lambda_{2}\right) \pm \Phi_{m n}\left(\Lambda_{1}, \Lambda_{2}\right)}{\sqrt{2\left(1+\delta_{12}\right)}}\left|V\left(R, \Lambda_{1}, \Lambda_{2}\right)\right| \frac{\Phi m n^{\prime}(\Lambda 1, \Lambda 2) \pm \Phi m n^{\prime}\left(\Lambda_{1}, \Lambda_{2}\right)}{\sqrt{2\left(1+\delta^{\prime}{ }_{12}\right)}}\right\rangle \\
& =\frac{1}{2 \sqrt{\left(1+\delta^{\prime}{ }_{12}\right)\left(1+\delta_{12}\right)}}\left(\Phi_{m n}\left(\Lambda_{1}, \Lambda_{2}\right)\left|V\left(R, \Lambda_{1}, \Lambda_{2}\right)\right|\left|\Phi_{m n^{\prime}}\left(\Lambda_{1}, \Lambda_{2}\right)\right\rangle\right. \\
& +\left\langle\widetilde{\Phi}_{m n}\left(\Lambda_{1}, \Lambda_{2}\right)\left|V\left(R, \Lambda_{1}, \Lambda_{2}\right)\right| \widetilde{\Phi}_{m n^{\prime}}\left(\Lambda_{1}, \Lambda_{2}\right)\right\rangle \\
& \pm\left\langle\Phi_{m n}\left(\Lambda_{1}, \Lambda_{2}\right)\left|V\left(R, \Lambda_{1}, \Lambda_{2}\right)\right| \Phi_{m n^{\prime}}\left(\Lambda_{1}, \Lambda_{2}\right)\right\rangle \\
& \left. \pm\left\langle\widetilde{\Phi}_{m n}\left(\Lambda_{1}, \Lambda_{2}\right)\left|V\left(R, \Lambda_{1}, \Lambda_{2}\right)\right| \Phi_{m n^{\prime}}\left(\Lambda_{1}, \Lambda_{2}\right)\right\rangle\right)
\end{aligned}
$$

The next step is to take into account that with our choice of coordinates the value of the potential does not change under the swap of two molecules. (Note that this is not necessarily the case with other choices of coordinates, see Appendix A. $)$ So, using $V\left(R, \Lambda_{1}, \Lambda_{2}\right)=V\left(R, \tilde{\Lambda}_{2}, \tilde{\Lambda}_{1}\right)$ and $\tilde{\Phi}_{m n}\left(\Lambda_{1}, \Lambda_{2}\right)=$ $\Phi_{m n}\left(\tilde{\Lambda}_{2}, \tilde{\Lambda}_{1}\right)=p \Phi_{m \tilde{n}}\left(\Lambda_{1}, \Lambda_{2}\right)$, eq 19 , we can group terms in the previous expression as follows:

$$
\begin{aligned}
& M_{n^{\prime}}^{n( \pm)}(R)=\frac{1}{2 \sqrt{\left(1+\delta_{12}^{r}\right)\left(1+\delta_{12}\right)}}\left(\left\langle\Phi_{m n}\left(\Lambda_{1} \Lambda_{2}\right)\right)\left|V\left(R, \Lambda_{1}, \Lambda_{2}\right)\right| \Phi_{m n^{\prime}}\left(\Lambda_{v} \Lambda_{2}\right)\right\rangle \\
& +\left\langle\Phi_{m m}\left(\tilde{\Lambda}_{2} \tilde{\Lambda}_{1}\right)\left|V\left(R, \tilde{\Lambda}_{2}, \tilde{\Lambda}_{1}\right)\right| \Phi_{m n^{\prime}}\left(\tilde{\Lambda}_{2}, \tilde{\Lambda}_{1}\right)\right\rangle \pm p^{\prime}\left\langle\Phi_{m m}\left(\Lambda_{1}, \Lambda_{2}\right)\left|V\left(R, \Lambda_{1}, \Lambda_{2}\right)\right| \Phi_{m \tilde{n}^{\prime}}\left(\Lambda_{1}, \Lambda_{2}\right)\right\rangle \\
& \left. \pm p^{\prime}\left\langle\Phi_{m m}\left(\tilde{\Lambda}_{2} \tilde{\Lambda}_{1}\right)\left|V\left(R, \tilde{\Lambda}_{2} \tilde{\Lambda}_{1}\right)\right| \Phi_{m \tilde{n}^{\prime}}\left(\tilde{\Lambda}_{2} \tilde{\Lambda}_{1}\right)\right\rangle\right) \\
& =\frac{1}{\sqrt{\left(1+\delta_{12}^{r}\right)\left(1+\delta_{12}\right)}}\left(\left\langle\Phi_{m m}\left(\Lambda_{y} \Lambda_{2}\right)\right)\left|V\left(R, \Lambda_{1} \Lambda_{2}\right)\right| \Phi_{m m^{\prime}}\left(\Lambda_{\nu}, \Lambda_{2}\right)\right\rangle \\
& \left. \pm p^{\prime}\left\langle\Phi_{m m}\left(\Lambda_{y}, \Lambda_{2}\right)\left|V\left(R, \Lambda_{1}, \Lambda_{2}\right)\right| \Phi_{m \tilde{n}}\left(\Lambda_{y}, \Lambda_{2}\right)\right\rangle\right) \\
& M_{n^{\prime}}^{n( \pm)}(R) \\
& =\frac{1}{2 \sqrt{\left(1+\delta^{\prime}{ }_{12}\right)\left(1+\delta_{12}\right)}}\left(\left\langle\Phi_{m n}\left(\Lambda_{1}, \Lambda_{2}\right)\right)\left|V\left(R, \Lambda_{1}, \Lambda_{2}\right)\right| \Phi m n^{\prime}\left(\Lambda_{1}, \Lambda_{2}\right)\right\rangle 2 \\
& +\left\langle\Phi_{m n}\left(\tilde{\Lambda}_{2}, \tilde{\Lambda}_{1}\right)\left|V\left(R, \tilde{\Lambda}_{2}, \tilde{\Lambda}_{1}\right)\right| \Phi_{m n^{\prime}}\left(\tilde{\Lambda}_{2}, \tilde{\Lambda}_{1}\right)\right\rangle \\
& \pm p^{\prime}\left\langle\Phi_{m n}\left(\Lambda_{1}, \Lambda_{2}\right)\left|V\left(R, \Lambda_{1}, \Lambda_{2}\right)\right| \Phi_{m \tilde{n}^{\prime}}\left(\Lambda_{1}, \Lambda_{2}\right)\right\rangle \\
& \left. \pm p^{\prime}\left\langle\Phi_{m n}\left(\tilde{\Lambda}_{2}, \tilde{\Lambda}_{1}\right)\left|V\left(R, \tilde{\Lambda}_{2}, \tilde{\Lambda}_{1}\right)\right| \Phi_{m \tilde{n}^{\prime}}\left(\tilde{\Lambda}_{2}, \tilde{\Lambda}_{1}\right)\right\rangle\right) \\
& =\frac{1}{2 \sqrt{\left(1+\delta^{\prime}{ }_{12}\right)\left(1+\delta_{12}\right)}}\left(\left\langle\Phi_{m n}\left(\Lambda_{1}, \Lambda_{2}\right)\right)\left|V\left(R, \Lambda_{1}, \Lambda_{2}\right)\right| \Phi_{m n^{\prime}}\left(\Lambda_{1}, \Lambda_{2}\right)\right\rangle \\
& \left. \pm p^{\prime}\left\langle\Phi_{m n}\left(\Lambda_{1}, \Lambda_{2}\right)\left|V\left(R, \Lambda_{1}, \Lambda_{2}\right)\right| \Phi_{m \tilde{n}^{\prime}}\left(\Lambda_{1}, \Lambda_{2}\right)\right\rangle\right)
\end{aligned}
$$


where state $\tilde{n}^{\prime}=\left\{f^{\prime} n_{2}{ }^{\prime} n_{1}{ }^{\prime}\right\}$ is obtained from $n^{\prime}=\left\{f^{\prime} n_{1}{ }^{\prime} n_{2}{ }^{\prime}\right\}$ by swapping particles one and two.

Note that in eq 23, we employed the following properties:

$$
\begin{aligned}
& \left\langle\Phi_{m n}\left(\Lambda_{\nu} \Lambda_{2}\right)\left|V\left(R, \Lambda_{1}, \Lambda_{2}\right)\right| \Phi_{m n^{\prime}}\left(\Lambda_{1}, \Lambda_{2}\right)\right\rangle=\left\langle\Phi_{m u}\left(\tilde{\Lambda}_{\nu} \tilde{\Lambda}_{1}\right)\left|V\left(R, \tilde{\Lambda}_{v}, \tilde{\Lambda}_{1}\right)\right| \Phi_{m n^{\prime}}\left(\tilde{\Lambda}_{2}, \tilde{\Lambda}_{1}\right)\right\rangle \\
& \left\langle\Phi_{m n}\left(\Lambda_{1}, \Lambda_{2}\right)\left|V\left(R, \Lambda_{1}, \Lambda_{2}\right)\right| \Phi_{m \tilde{n}}\left(\Lambda_{1}, \Lambda_{2}\right)\right\rangle=\left\langle\Phi_{m u}\left(\tilde{\Lambda}_{v}, \tilde{\Lambda}_{1}\right)\left|V\left(R, \tilde{\Lambda}_{v}, \tilde{\Lambda}_{1}\right)\right| \Phi_{m \tilde{n}}\left(\tilde{\Lambda}_{v}, \tilde{\Lambda}_{1}\right)\right\rangle \\
& \left\langle\Phi_{m n}\left(\Lambda_{1}, \Lambda_{2}\right)\left|V\left(R, \Lambda_{1}, \Lambda_{2}\right)\right| \Phi m n^{\prime}\left(\Lambda_{1}, \Lambda_{2}\right)\right\rangle \\
& =\left\langle\Phi_{m n}\left(\tilde{\Lambda}_{2}, \tilde{\Lambda}_{1}\right)\left|V\left(R, \tilde{\Lambda}_{2}, \tilde{\Lambda}_{1}\right)\right| \Phi_{m n^{\prime}}\left(\tilde{\Lambda}_{2}, \tilde{\Lambda}_{1}\right)\right\rangle \\
& \left\langle\Phi_{m n}\left(\Lambda_{1}, \Lambda_{2}\right)\left|V\left(R, \Lambda_{1}, \Lambda_{2}\right)\right| \Phi_{m \tilde{n}^{\prime}}\left(\Lambda_{1}, \Lambda_{2}\right)\right\rangle \\
& =\left\langle\Phi_{m n}\left(\tilde{\Lambda}_{2}, \tilde{\Lambda}_{1}\right)\left|V\left(R, \tilde{\Lambda}_{2}, \tilde{\Lambda}_{1}\right)\right| \Phi_{m \tilde{n}^{\prime}}(\Lambda 2, \Lambda 1)\right\rangle
\end{aligned}
$$

One can compute nonzero matrix elements using eq 23 directly, building the basis of symmetrized functions. Alternatively, if the total state-to-state transition matrix (without taking into account the exchange symmetry) is computed as outlined in section II-C and Appendix B, then the symmetrized matrix element for states of given parity can be easily constructed by superposition:

$$
\begin{gathered}
M_{n^{\prime}}^{\mathrm{n}( \pm)}=\left(M_{\mathrm{n}^{\prime}}^{\mathrm{n}} \pm p^{\prime} M_{n^{\prime}}^{\mathrm{n}}\right) / \sqrt{\left(1+\delta_{12}\right)\left(1+\delta_{12}^{\prime}\right)} \\
M_{n^{\prime}}^{n( \pm)}=\left(M_{n^{\prime}}^{n} \pm p^{\prime} M_{\tilde{n}^{\prime}}^{n}\right) \sqrt{\left(1+\delta_{12}\right)\left(1+\delta_{12}^{\prime}\right)}
\end{gathered}
$$

It is possible to show that when the parities are different for the initial and the final states of the system, the matrix element is zero:

$$
\begin{aligned}
& M_{M^{\prime}}^{\prime \prime}(R) \\
& =\left\langle\Phi_{m n}^{ \pm}\left(\Lambda_{1}, \Lambda_{2}\right)\left|V\left(R, \Lambda_{v} \Lambda_{2}\right)\right| \Phi_{m n^{\prime}}^{\mp}\left(\Lambda_{v} \Lambda_{2}\right)\right\rangle \\
& =\frac{1}{2 \sqrt{\left(1+\delta_{12}^{\prime}\right)\left(1+\delta_{12}\right)}}\left(\left\langle\Phi_{m m}\left(\Lambda_{v}, \Lambda_{2}\right)\left|V\left(R, \Lambda_{1}, \Lambda_{2}\right)\right| \Phi_{m n}\left(\Lambda_{1}, \Lambda_{2}\right)\right\rangle-\left\langle\Phi_{m m}\left(\tilde{\Lambda}_{v} \tilde{\Lambda}_{1}\right)\left|V\left(R, \tilde{\Lambda}_{2}, \tilde{\Lambda}_{1}\right)\right|\right.\right. \\
& \left.\Phi_{m n}\left(\tilde{\Lambda}_{2} \tilde{\Lambda}_{1}\right)\right\rangle \pm p^{\prime}\left\langle\Phi_{m m}\left(\Lambda_{y} \Lambda_{2}\right)\left|V\left(R, \Lambda_{1}, \Lambda_{2}\right)\right| \Phi_{m \tilde{n}^{\prime}}\left(\Lambda_{y}, \Lambda_{2}\right)\right\rangle \\
& \left.\mp p^{\prime}\left\langle\Phi_{m v}\left(\tilde{\Lambda}_{2}, \tilde{\Lambda}_{1}\right)\left|V\left(R, \tilde{\Lambda}_{2} \tilde{\Lambda}_{1}\right)\right| \Phi_{m \tilde{\pi}^{\prime}}\left(\tilde{\Lambda}_{2}, \tilde{\Lambda}_{1}\right)\right\rangle\right)=0
\end{aligned}
$$




$$
\begin{aligned}
& M_{n^{\prime}}^{n( \pm)}=\left\langle\Phi_{m n}^{ \pm}\left(\Lambda_{1}, \Lambda_{2}\right)\left|V\left(R, \Lambda_{1}, \Lambda_{2}\right)\right| \Phi_{m n^{\prime}}^{\mp}\left(\Lambda_{1}, \Lambda_{2}\right)\right\rangle \\
& =\frac{1}{2 \sqrt{\left(1+\delta^{\prime}{ }_{12}\right)\left(1+\delta_{12}\right)}}\left(\left\langle\Phi_{m n}\left(\Lambda_{1}, \Lambda_{2}\right)|V(R,)| \Phi_{m n^{\prime}}\left(\Lambda_{1}, \Lambda_{2}\right)\right\rangle\right. \\
& -\left\langle\Phi_{m n}\left(\tilde{\Lambda}_{2}, \tilde{\Lambda}_{1}\right)\left|V\left(R, \tilde{\Lambda}_{2}, \tilde{\Lambda}_{1}\right)\right| \Phi_{m n^{\prime}}\left(\tilde{\Lambda}_{2}, \tilde{\Lambda}_{1}\right)\right\rangle \\
& \pm p^{\prime}\left\langle\Phi_{m n}\left(\Lambda_{1}, \Lambda_{2}\right)\left|V\left(R, \Lambda_{1}, \Lambda_{2}\right)\right| \Phi_{m \tilde{n}^{\prime}}\left(\Lambda_{1}, \Lambda_{2}\right)\right\rangle \\
& \left.\mp p^{\prime}\left\langle\Phi_{m n}\left(\tilde{\Lambda}_{2}, \tilde{\Lambda}_{1}\right)\left|V\left(R, \tilde{\Lambda}_{2}, \tilde{\Lambda}_{1}\right)\right| \Phi_{m \tilde{n}^{\prime}}\left(\tilde{\Lambda}_{2}, \tilde{\Lambda}_{1}\right)\right\rangle\right)=0
\end{aligned}
$$

II-F Effect of Potential Symmetry for $\mathrm{H}_{2} \mathrm{O}$ Molecules

Several properties of the potential energy surface expansion coefficients in eq 11 are worth mentioning:(37,38)

$$
\begin{aligned}
& V_{l_{n_{1}} I_{1} \eta_{1} l}=(-1)^{l_{1}+l_{2}} V_{l_{n_{1}} l_{n_{1}} l} \\
& V_{l_{1} \eta_{1} l_{2} \eta_{2} l}=(-1)^{l_{1}+l_{2}} V_{l_{2} \eta_{2} l_{1} \eta_{1} l} \\
& V_{n_{\eta_{1} i_{1} \eta_{1} l} l}=(-1)^{l_{1}+\eta_{1}+l_{2}+\eta_{2}+l} V_{\mathrm{I}_{1}-\eta_{1} l_{1}-\eta_{1} l} \\
& V_{l_{1} \eta_{1} l_{2} \eta_{2} l}=(-1)^{l_{1}+n_{1}+l_{2}+n_{2}+l} V_{l_{1}-\eta_{2} l_{2}-\eta_{2} l} \\
& V_{l_{n_{1} I_{1} \eta_{2} l}}=0 \quad \text { for odd } \eta_{1,2} \\
& V_{l_{1} \eta_{1} l_{2} \eta_{2} l}=0 \text { for odd } \eta_{1,2}
\end{aligned}
$$

The first of these is related to the fact that two collision partners are identical and swapping them does not change the potential energy. The meaning of the second is that the potential should remain the same under simultaneous inversion of both molecules. The third reflects the $C_{2 v}$ symmetry of each water molecule.

In our calculations reported in the next section, we included in the expansion of eq 11 all terms with $I \leq 6 .(37,38)$ This is, roughly, 2000 terms total in the PES expansion. Using the properties 
of eqs 25 and 26 , this number is reduced to just 254 nonzero and unique terms, with $l_{1,2} \leq 6$ and even values of $\eta_{1,2}$.

\section{Results}

\section{III-A Properties of Potential and Matrix Elements}

It is expected that a system of two polar water molecules would exhibit a rather strong long-range dipole-dipole interaction. This property of the potential energy surface becomes clear if we analyze the behavior of the expansion coefficients $V_{h} \eta_{1} l_{2} \eta_{2} l(R)$ in eq 11, as a function of molecule-molecule distance $R$. The expansion coefficients have been computed numerically by projecting the original potential on the set of real functions from eq 12. The integration has been performed on a fivedimensional Gauss-Legendre quadrature point grid, where the size of the grid has been defined by convergence studies and set to 20 for integration along each Euler angle. Several most important coefficients are presented in Figure 2, labeled by five numbers: $l_{1} \eta_{1} l_{2} \eta_{2} l$. Recall that $\eta_{1}$ and $\eta_{2}$ are even, while $I_{1}$ and $I_{2}$ are such that $\left|I_{1}-I_{2}\right| \leq I \leq I_{1}+I_{2}$. The dipole-dipole interaction term corresponds to $I_{1}=I_{2}=1\left(I=2\right.$ and $\left.\eta_{1}=\eta_{2}=0\right)$; so, it is labeled by 10102 . From Figure 2 we see that this term is negative and large. At a distance of $R=20 a_{0}$ (about $10 \AA$ ), its value is about -2.7 $\mathrm{kcal} / \mathrm{mol}$. At a distance of $R=40 a_{0}$, its value is still non-negligible, about $-0.34 \mathrm{kcal} / \mathrm{mol}$. We checked and found that in the asymptotic range the expected dipole-dipole behavior, $V_{10102} \sim 1 / R^{3}$, is well satisfied. Interestingly, from Figure 2 we see that the magnitude of $V_{10102}$ is larger than that of the isotropic (elastic scattering) term $V_{00000}$, through the entire relevant range of distances $R$. The well depth of $V_{00000}$ is about $30 \mathrm{kcal} / \mathrm{mol}$, with a minimum energy point located near $R \sim 6.5 a_{0}$.

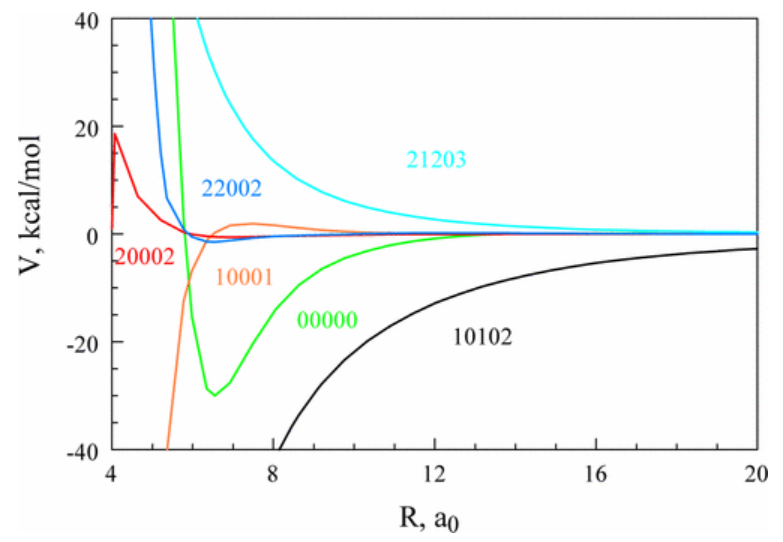

Figure 2. Expansion coefficients, as a function of molecule-molecule distance, for the potential energy surface of the water-water system represented by eq 11. The six most important terms are shown. Labels include five indices: $l_{1} \eta_{1} l_{2} \eta_{2} l$. The curves are color-coded, accordingly. Note that the dipole-dipole interaction term (black) exceeds the elastic scattering term (green), at all relevant distances.

Several other most important dipole-like $\left(l_{1}=1\right)$ and quadrupole-like $\left(l_{1}=2\right)$ terms are also presented in Figure 2. They all are smaller than the dipole-dipole term $V_{10102}$ and vanish faster as the molecule-molecule separation increases. Still, some of them are comparable in magnitude to the elastic term $V_{00000}$. This means that the PES is highly anisotropic, even at large distances. The data file with $R$-dependence of 254 expansion coefficients we used in this work is included in the Supporting Information. 
As we are going to demonstrate below, one important consequence of the long-range anisotropy of the PES is that scattering calculations for the water-water system must start from very large initial molecule-molecule distances and must include very large values of the impact parameter. Of course, these numbers depend somewhat on collision energy, but in any case are unusually large. For example, for a collision energy of $E=1500 \mathrm{~cm}^{-1}$ we had to take $R_{\max } \sim 100 a_{0}$ and $b_{\max } \sim 30 a_{0}$ in order to guarantee convergence of integral inelastic scattering cross sections (for excitation of several lower lying states, starting from the ground state of the system) within $0.5 \%$. The situation is even worse for the elastic scattering channels (see below), where accurate treatment of the scattering phase in the asymptotic range is essential for convergence.

Dominance of the dipole-dipole interaction is further reflected by state-to-state transition matrix elements $M_{n^{\prime \prime}}( \pm)$, computed as outlined in section II and Appendix B. Here it becomes convenient to switch from state labeling employed above $\left(n_{1}=\left\{j_{1} k_{1}{ }^{A} k_{1} C\right\}, n_{2}=\left\{j_{2} k_{2} A k_{2} C\right\}\right.$, and $\left.n=\left\{j n_{1} n_{2}\right\}\right)$ to the standard labeling that uses subscripts: $j_{1 k_{1}}{ }^{A} k_{1} C j_{2 k 2}{ }^{A} k_{2} C(j m)$. For example, the ground states of two molecules are labeled as $0_{00}$ and $0_{00}$, which gives total $j=0$ and $m=0$ or if we combine everything, $0_{00} 0_{00}(00)$.

Now consider the excitation of the state $1_{11}$ in one of two (identical) molecules. Since $\left|j_{1}-j_{2}\right|$ $\leq j \leq j_{1}+j_{2}$ we have to include, into the basis set expansion of eq 8 , the excited total $j=1$ with its associated states $m=0, \pm 1$. Since the initial state $0_{00} 0_{00}(00)$ has $m=0$ and since matrix $M_{n^{\prime \prime}}( \pm)$ is diagonal in $m$, we obtain a nonzero transition element only for the final state $1_{11} 0_{00}(10)$. However, the final states with $m= \pm 1$ will receive population from the state with $m=0$ due to the Coriolis coupling effect, $\Delta m= \pm 1$; see eq 4. Thus, we have to include the elastic (diagonal) matrix elements $M_{n^{\prime \prime}}( \pm)$ for these states as well, namely, $1_{11} 0_{00}(11)$. Note that the value of the matrix element is the same for positive and negative values of projection $m$ of the total $j$.

Similarly, for excitation of the state $1_{11}$ in both molecules, we include total $j=0,1$, and 2 . If the initial state is the ground state with $m=0$, then nonzero transition matrix elements $M_{n^{\prime}}( \pm)$, due to potential coupling, should be included just for the following states (all with final $m=0$ ): $1_{11} 1_{11}(00)$, $1_{11} 1_{11}(10)$, and $1_{11} 1_{11}(20)$. Coriolis coupling then would populate $1_{11} 1_{11}(11), 1_{11} 1_{11}(21)$, and finally $1_{11} 1_{11}(22)$.

Next, for the excitation of state $22_{02}$ in one molecule only, the state $2_{02} 0_{00}(20)$ will have nonzero transition matrix elements $M_{n^{\prime}} n( \pm)$ due to potential coupling. State $2_{02} 0_{00}(21)$ and eventually $2{ }_{02} 0_{00}$ (22) would be populated by Coriolis coupling and so on. In Figure 3 we not only present matrix elements, as a function of $R$, for state-to-state transitions from the ground state of the system $0_{00} 0_{00}(00)$ to several most important excited states of positive exchange parity but also the diagonal matrix elements for those excited states, important for the elastic scattering processes. The dipoledipole interaction manifests here, first of all, through the largest matrix element for excitation of the state $1_{11} 1_{11}(20)$, starting from the ground state $0_{00} 0_{00}(00)$. The value of this matrix element decays slowly at large distances (lower black curve in Figure 3 ). For example, at $R=20 a_{0}$ its value is still significant, about $-12 \mathrm{~cm}^{-1}$. Even a distance of $R=40 a_{0}$ it is still non-negligible, about $-1.5 \mathrm{~cm}^{-1}$. Another consequence of a large dipole-dipole interaction term is that the values of diagonal matrix elements for the elastic scattering of the states $1_{11} 0_{00}(10)$ and $1_{11} 0_{00}(11)$ are also large and decay slowly (two red curves in Figure 3). Indeed, the direct effect of the dipole-dipole potential term $V_{10102}$ on the states $1_{11} 0_{00}$ can be explicitly demonstrated analytically using eqs 11 and $\underline{25}$. 


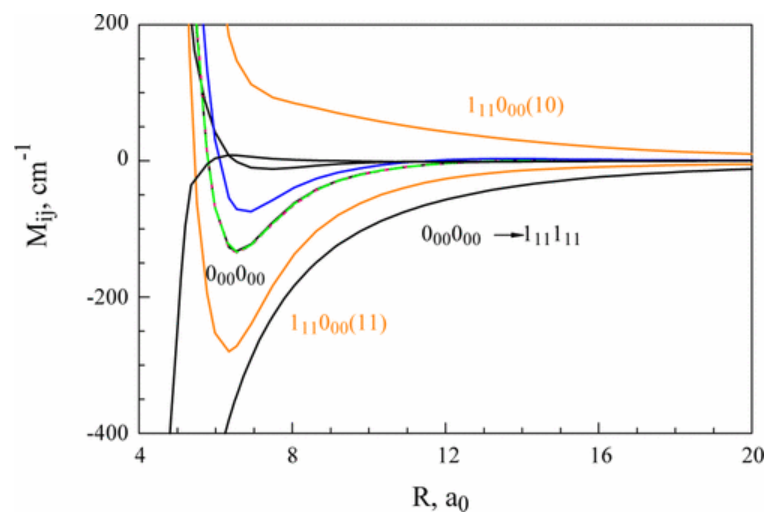

Figure 3. State-to-state transition matrix elements as a function of water-water distance $R$. Black curves correspond to transitions from the ground state $0_{00} 0_{00}(00)$ to the following final states (including the elastic channel): $0_{00} 0_{00}(00), 1_{11} 0_{00}(10), 1_{11} 1_{11}(20)$, and $2_{02} 0_{00}(20)$. Orange curves, upper and lower, correspond to elastic scattering for $1_{11} 0_{00}(10)$ and $1_{11} 0_{00}(11)$ states, respectively. The blue curve is for the elastic scattering off the state $2_{02} 0_{00}(20)$. Dashed green and magenta curves are for the elastic scattering off states $1_{11} 1_{11}(20)$ and $1_{11} 1_{11}(21)$, respectively (note that they nearly coincide with the elastic $0_{00} 0_{00}$ term).

Thus, in the following sections the focus is on the inelastic transition to the $1_{11} 1_{11}$ state (from the ground state $0_{00} 0_{00}$ ) and on elastic scattering off $1_{11} 0_{00}$, since both processes are driven by strong dipole-dipole components of the interaction potential.

\section{III-B Inelastic Scattering Off the Ground State}

Within the MQCT framework and using the potential expansion outlined above, we carried out inelastic scattering calculations for the water-water system. Sampling of the initial conditions for collision, propagation of the mixed quantum/classical trajectories, calculations of the final transition probabilities, state-to-state cross sections, and channel-to-channel cross sections (summed over the final and averaged over the initial degenerate states) follow the procedures outlined in our earlier papers (27-29) and will not be reviewed again for the purpose of brevity.

Here we only consider excitation of the ground state $0_{00} 0_{00}$. Since initially the internal rotational angular momentum is null $(j=0$ and $m=0$ ), the value of orbital angular momentum l (treated classically within the MQCT) is equal to the grand angular momentum Jof the system. Its maximum value $J_{\max }$ is a convergence parameter in the MQCT calculations, just as in the full-quantum scattering calculations. The value of $J_{\max }\left(\right.$ and $l_{\max }$ ) depends somewhat on collision energy. For example, at $E=500,1500$, and $5000 \mathrm{~cm}^{-1}$ we used $J_{\max }=380,660$, and 1100 , respectively. These numbers are rather large, by quantum mechanical standards. They reflect a rather heavy reduced mass of the system, and a long-range nature of the dipole-dipole interaction. In practice, instead of energy-dependent $J_{\max }$, it is more convenient to specify the maximum value of the impact parameter $b_{\max }$, since it is basically independent of collision energy (just weakly dependent in the considered energy range). As mentioned in the previous section, the value $b_{\max } \sim 30 a_{0}$ was used (together with $R_{\max } \sim 100 a_{0}$ ).

Another convergence parameter of the MQCT calculations is the number of trajectories used to sample orbital angular momentum in the range from $l=0$ (head-on collision, back scattering) to $l_{\max }$ (forward scattering in the asymptotic range with vanishing transition probability). Here the relevant range of $l$ as sampled continuously (noninteger values) and uniformly (using an 
equidistant grid of points in the range $0 \leq l \leq l_{\max }$ ). These values of $l$ define initial conditions for a batch of independent MQCT trajectories. We found that 60 trajectories were typically sufficient to represent relatively smooth opacity functions in the considered energy range. Figure 4 gives an example of opacity functions (transition cross section vs impact parameter) for the dipole-driven transition $0_{00} 0_{00} \rightarrow 1_{11} 1_{11}$ at collision energy of $E=1500 \mathrm{~cm}^{-1}$. Note that our opacity functions include a factor of $(2 J+1)$ for each trajectory, to properly reflect the importance of the process in the overall cross section. The results of two calculations are presented in Figure 4, one obtained with $R_{\max }=50 a_{0}$ and the other with $R_{\max }=100 a_{0}$. This emphasizes that due to the long-range dipole-dipole interaction the initial value of the molecule-molecule distance in scattering calculations must be rather large in order to obtain well converged results. A seemingly large value of $R_{\max }=50 a_{0}$ appears to be insufficient, since it not only leads to nonzero transition probability at large impact parameters but also to some deviations of the transition probability in the entire range of impact parameters. The value of $R_{\max }=100 a_{0}$ is large enough. The difference of integral inelastic cross sections in these two cases is close to $5 \%$.

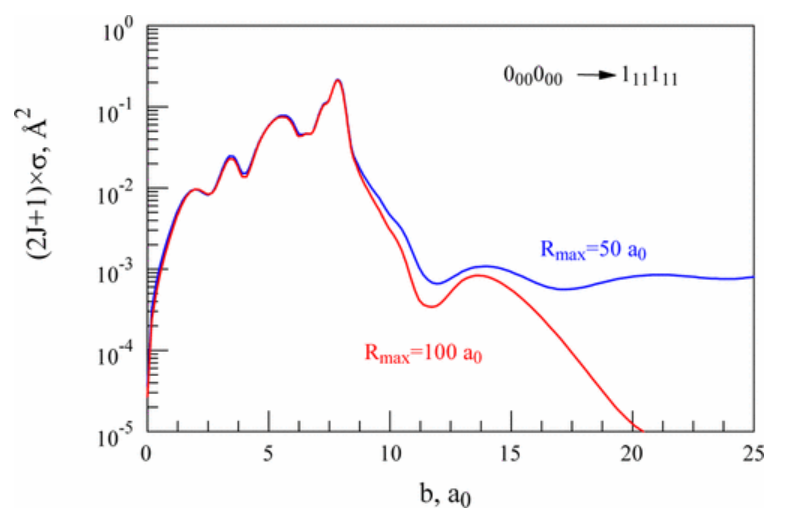

Figure 4. Demonstration of the effect of long-range dipole-dipole interaction onto the opacity function for excitation of $1_{11} 1_{11}$ (starting from the ground state $0_{00} 0_{00}$ ) through the choice of the initial value of the molecule-molecule distance $R_{\max }$ for scattering calculations. A seemingly large value of $R_{\max }=50 a_{0}$ appears to be insufficient, since it not only leads to nonzero transition probability at large impact parameters but also to some deviations of the transition probability in the entire range of impact parameters. The value of $R_{\max }=100 a_{0}$ is large enough for the description of $\mathrm{H}_{2} \mathrm{O}+\mathrm{H}_{2} \mathrm{O}$.

Figure 5 represents examples of the opacity functions for three important transitions at various collision energies. The upper frame corresponds to the dipole-driven transition $0_{00} 0_{00} \rightarrow 1_{11} 1_{11}$. The second frame corresponds to the excitation of $2_{02} 0_{00}$, which (mostly) receives its population indirectly through the state $1_{11} 1_{11}$. The lower frame corresponds to the excitation of $1_{11} 0_{00}$, which can be characterized as a nondipole driven and thus is much weaker. Different curves in each frame show how the opacity function evolves as collision energy is changed. Here one can identify, very clearly, a presence of two scattering regimes. One corresponds to lower collision energies and lower impact parameters (e.g., $E \sim 1000 \mathrm{~cm}^{-1}$ and $4 \leq b \leq 7 a_{0}$ ). This is a typical behavior seen in many inelastic scattering calculations in the past, on many molecules, but in addition to this, the dipole-driven transition to $1_{11} 1_{11}$ demonstrates very large probabilities at high collision energies and large impact parameters, simultaneously (e.g., $E \sim 10000 \mathrm{~cm}^{-1}$ and $b \sim 13 a_{0}$ ). The same effect translates into a consecutive excitation of $2_{02} 0_{00}$ (from $1_{11} 1_{11}$ ). This interesting feature is not typical and is the result of a long-range rather strong dipole-dipole interaction. 

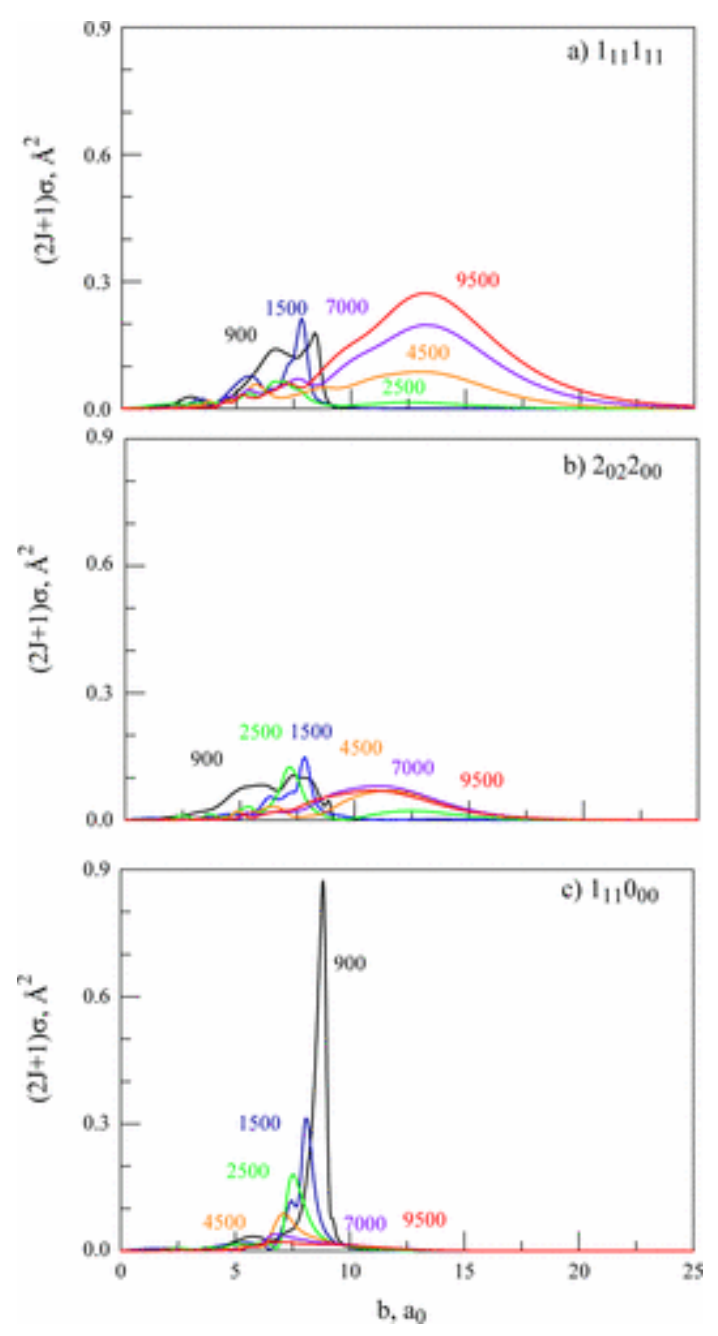

Figure 5. Opacity functions for three inelastic scattering processes, starting from the ground state $0_{00} 0_{00}$. Collision energies are indicated in the figure. Three frames correspond to the following final states of $\mathrm{H}_{2} \mathrm{O}+\mathrm{H}_{2} \mathrm{O}$ : (a) $1_{11} 1_{11}$, (b) $22_{02} 0_{00}$, and (c) $1_{11} 0_{00}$. See text for discussion.

The last convergence parameter in the MQCT calculations is the number of internal rotational states in the basis set. Here we included all states with $j_{1} \leq 6, j_{2} \leq 6$, and $j \leq 8$ such that $\left|j_{1}-j_{2}\right| \leq j \leq j_{1}+j_{2}$. All nondegenerate components $\left\{k_{1}{ }^{A} k_{1} C\right\}$ and $\left\{k_{2}^{A} k_{2} C\right\}$ of these $j_{1}$ and $j_{2}$ states were included, which resulted in 132 nondegenerate levels, or scattering channels, all listed in the Supporting Information. Energies of these levels cover confidently the range up to $700 \mathrm{~cm}^{-1}$, and some of them reach $990 \mathrm{~cm}^{-1}$. With this basis set, cross sections for excitations of 15 lower energy channels are converged within 5\%, for collision energy $E=1500 \mathrm{~cm}^{-1}$. For lower scattering energies, this basis set is certainly sufficient, but even for higher scattering energies (here we considered up to 10000 $\mathrm{cm}^{-1}$ ), the upper states of the basis receive very little populations, which means that results remain reasonably accurate (conservative estimate is within 10-15\%). Note that we carried out the fully coupled version of the MQCT calculations, without the CS approximation (see eq 5 and the discussion just below it), including explicitly all degenerate components $m$ of considered $j \leq 8$ states, which resulted in 5932 states total in our calculations. 
In Figure 6 we plotted inelastic scattering cross sections as a function of collisional energy in the range from 100 to $10000 \mathrm{~cm}^{-1}$ for the 15 most important excited states. Figure 6a shows data for the excitation of five lower lying rotational states: $1_{11} 0_{00}, 1_{11} 1_{11}, 2_{02} 0_{00}, 2_{11} 0_{00}$, and $2_{20} 0_{00}$, whereas Figure $6 \mathrm{~b}$ shows the data for the remaining 10 states (all of them are labeled; all data are available in the Supporting Information). The range of confident prediction is to the right of the dashed line. For collision energies $500 \leq E \leq 1500 \mathrm{~cm}^{-1}$, cross sections are converged within $5 \%$ and within $10-15 \%$ in the range $1500 \leq E \leq 10000 \mathrm{~cm}^{-1}$.

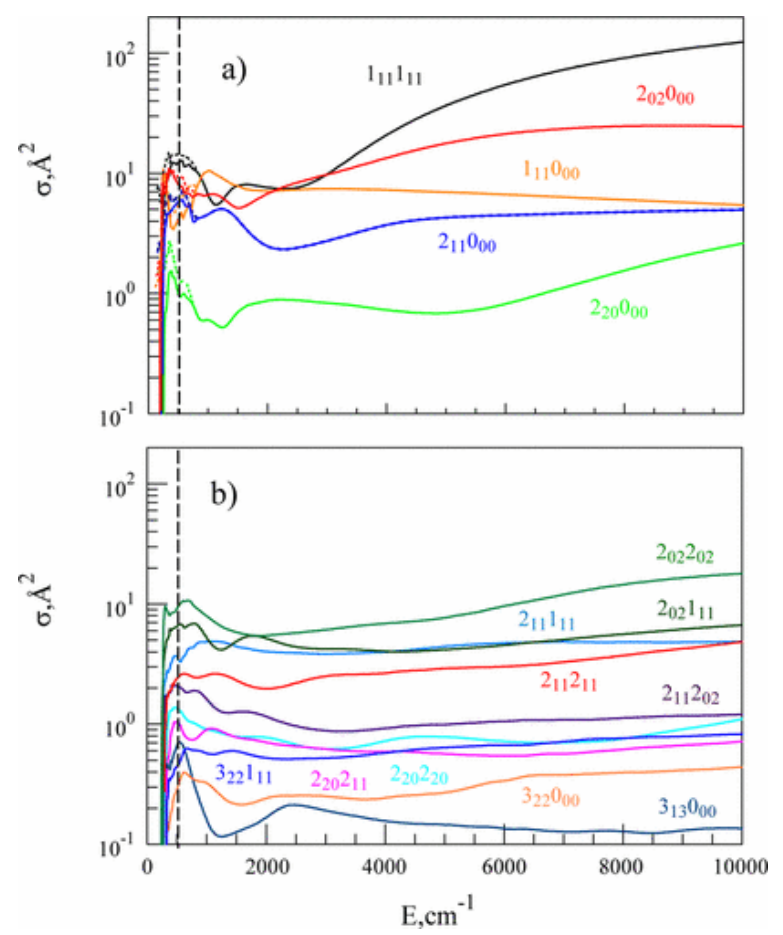

Figure 6. Inelastic scattering cross section as a function of collisional energy for $\mathrm{H}_{2} \mathrm{O}+\mathrm{H}_{2} \mathrm{O}$. All curves correspond to the excitation from the ground state $0_{00} 0_{00}$. The region to the left of the dashed line is found to contain scattering resonances, not treated here. The effect of orbiting trajectories is shown by dashed lines (see text for details). Frame a shows the excitation of five lower energy states. Frame b shows the excitation of the other 10 most important states. Convergence is within $5 \%$ for energies up to $1500 \mathrm{~cm}^{-1}$ and is within $10-15 \%$ at higher energies.

At collision energies $E<500 \mathrm{~cm}^{-1}$, to the left from the dashed line, we observed many orbiting trajectories, which is a vestige of quantum scattering resonances. It is not yet clear how to treat these cases. We removed orbiting trajectories if they make more than three loops to obtain nonresonant contribution to inelastic cross section. In the energy range where resonances are present, such data give nonresonant background of the overall energy dependence of a cross section. In Figure 6 these data are plotted by solid curves. They drop fast as the collision energy is decreased below $500 \mathrm{~cm}^{-1}$ because the number of orbiting trajectories in the batch also increases as the collision energy is reduced. For example, at energies $E \sim 200 \mathrm{~cm}^{-1}$ close to $30 \%$ of trajectories in the batch describe orbiting. As an experiment, we tried to stop orbiting trajectories after one period of rotation (roughly) and include them in the analysis. The resultant cross sections were significantly larger, compared to the nonresonant background, as shown in Figure 6 by dashed lines. A good recipe for analysis or resonant/orbiting trajectories within MQCT is yet to be found. At 
the present time, the range of confident MQCT predictions is restricted to higher collision energies, $E>500 \mathrm{~cm}^{-1}$. Hopefully, the full-quantum scattering calculations are affordable at lower energies and could be done 1 day for $E<500 \mathrm{~cm}^{-1}$. It would be interesting to compare those with our MQCT predictions at the matching point, somewhere near $E \sim 500 \mathrm{~cm}^{-1}$. It is likely that better understanding of orbiting trajectories, and their ties to scattering resonances, should be explored using simpler systems, such as $\mathrm{H}_{2} \mathrm{O}+\mathrm{He},(25)$ where broad isolated scattering resonances have been identified by the full-quantum calculations at low scattering energies.

High-energy behavior of the dipole-dipole driven excitation of the state $1_{11} 1_{11}$, seen in Figure $6 \mathrm{a}$, is quite surprising. One could expect that at higher collision energies the process of scattering is dominated by the repulsive short-range interaction and is limited to relatively small impact parameters. Normally, the long-range (usually weak and attractive) interaction dominates at low collision energies and large impact parameters. What we see here is neither case: the dipole-driven transition to $1_{11} 1_{11}$ is more intense at higher collision energies (see Figure 6a) and occurs mostly at large impact parameters (see Figure 5a). An explanation is found in Figures 2 and 3 , where we see that although the dipole-dipole term is indeed a long-range, it is not weak at all and, of course, is not purely attractive (it is anisotropic). This term contributes to the repulsive part of potential too and dominates scattering for all collision energies, and all impact parameters, in a somewhat unexpected way. As collision energy is increased from $E=1000$ to $10000 \mathrm{~cm}^{-1}$, the cross section for the excitation of $1_{11} 1_{11}$ increases by an order of magnitude and still keeps growing. The excitation of state $2{ }_{02} 0_{00}$ follows a similar trend, since it is populated through $1_{11} 1_{11}$. This is one interesting finding of this work, which may have important implications for the analysis of the nonequilibrium population of the rotational states of water (end emission of those) in astrophysical environments, such as star-forming regions, $(41,42)$ protostellar discs, $(43-45)$ or cometary comas. $(32,33)$ This aspect is also interesting mechanistically, since very few quantum scattering calculations of the dipole-dipole driven transitions are available at this point and are typically at lower collision energies.(46-48)

It is probably true to say that the full quantum scattering calculations for the $\mathrm{H}_{2} \mathrm{O}+\mathrm{H}_{2} \mathrm{O}$ system will be very expensive. One way to make those more affordable is to use the coupled-states (CS) approximation, which neglects the Coriolis coupling effect, but the accuracy of this approach is often hard to guarantee. Thus, we decided to test the accuracy of the CS-approach for $\mathrm{H}_{2} \mathrm{O}+\mathrm{H}_{2} \mathrm{O}$, relative to the fully coupled CC-approach, all within the MQCT framework. Figure 7 gives a comparison of the results obtained using the CC and CS methods. Typically, results of the approximate CS method deviate from the accurate CC method at low collision energies but converge to them at higher collision energies. The reason for this is that at low scattering energies the longrange interaction dominates (large impact parameter) and the Coriolis coupling is important, while at higher energies, the short-range interaction dominates (small impact parameters) and the Coriolis coupling is minor. What we see in Figure 7 is different. The results of two methods are in reasonable qualitative agreement within an order of magnitude, but there is no monotonic convergence of CS (dashed lines) toward CC (solid lines of the same color). Agreement is the best for excitation of states $1_{11} 1_{11}, 2_{02} 0_{00}$, and $2_{11} 0_{00}$, particularly at collision energies below $1000 \mathrm{~cm}^{-1}$, where the differences are on the order of $30-50 \%$. However, at higher collision energies the difference increases, reaching 200\% (a factor of 2). This can be explained by the fact that for the $\mathrm{H}_{2} \mathrm{O}+\mathrm{H}_{2} \mathrm{O}$ system large impact parameters remain important even at higher collision energies (due to dipole-dipole interaction, see Figure 5); thus, the Coriolis coupling is never minor. Larger differences seen for excitation of the state $2{ }_{11} 0_{00}$ have a different nature (specific to the MQCT 
method itself rather than to the CS-approximation). Overall, our conclusion here is that the CS approximation can be used only for a semiquantitative estimate of scattering cross sections, if the accuracy in the range of the factor of 2 is sufficient.

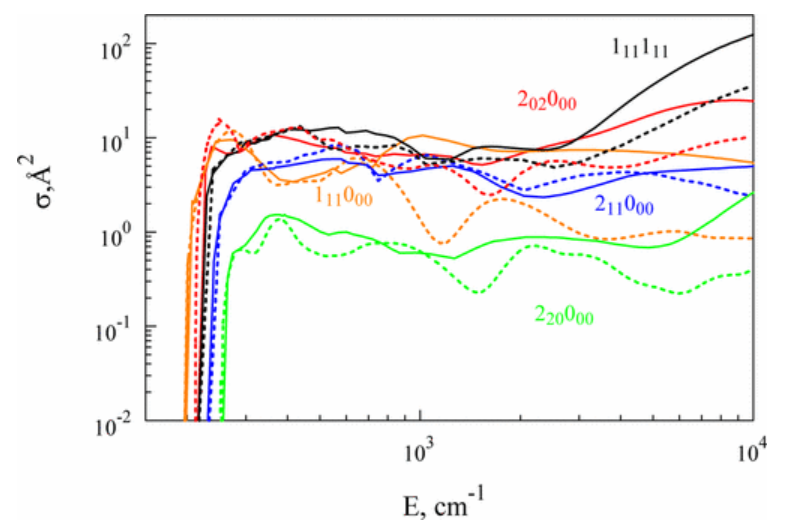

Figure 7. Test of the CS-approximation (dashed lines) relative to the fully coupled CC results (solid lines of the same color), all within the MQCT framework. Cross sections are shown for excitation of the same five states as in Figure 6 $\mathrm{a}$, starting from the ground state $0_{00} 0_{00}$. The same colors are used as in Figure 6a, but the collision energy (horizontal) is plotted using the log scale.

\section{III-C Most Important Elastic Scattering Channels}

As it was emphasized in the past publications, $(22,23,28)$ it is possible to restore the scattering phase within the MQCT approach, which enables rigorous calculations of differential cross sections, including that for the elastic scattering channel (impossible within the classical trajectory method). In Figure 8, we report the differential cross section for the elastic scattering off the ground state $0_{00} 0_{00}$ of the $\mathrm{H}_{2} \mathrm{O}+\mathrm{H}_{2} \mathrm{O}$ system for three values of scattering energies: 800,2500 , and $9500 \mathrm{~cm}^{-1}$. We found that in all cases the forward scattering dominates. For scattering at $800 \mathrm{~cm}^{-1}$, the amplitude is significant only within the range of $+0.6^{\circ}$. It shrinks to only $+0.3^{\circ}$ when the energy is raised to $9500 \mathrm{~cm}^{-1}$.

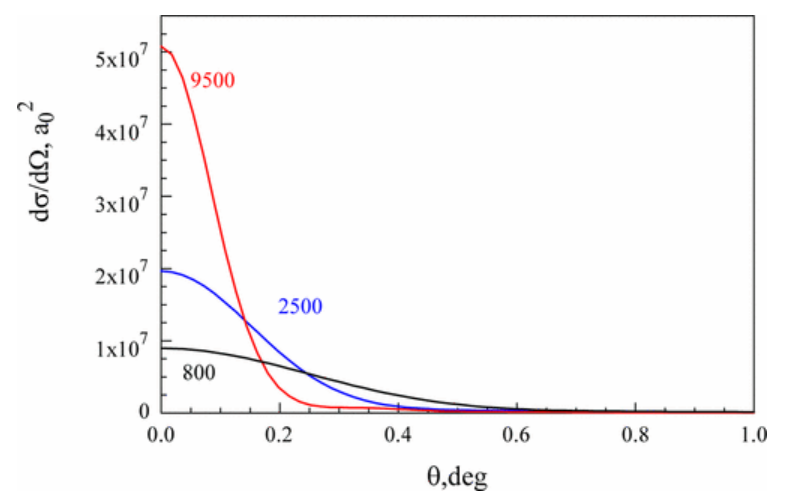

Figure 8. Differential (over scattering angle) cross section for the elastic channel of the $\mathrm{H}_{2} \mathrm{O}+\mathrm{H}_{2} \mathrm{O}$ system in the ground state $0_{00} 0_{00}$. Three values of collision energies are considered, as indicated in the picture: 800,2500 , and $9500 \mathrm{~cm}^{-1}$.

In Figure 9, we plotted the value of the integral cross section for the elastic scattering off the ground state $0_{00} 0_{00}$ as a function of collision energy and the same moiety for the exited level $1_{11} 0_{00}$, which, as explained above, is easily populated by the intense dipole-dipole driven transitions. Recall that 
this level contains three degenerate states of the total $j=1$, those with $m=0, \pm 1$ (see section III-A). Thus, we had to run two calculations: one with the initial state $1_{11} 0_{00}(10)$ and the other with the initial state $1_{11} 0_{00}(11)$. A separate calculation with $m=-1$ is not needed, since the results would be identical to that of $m=+1$, due to the symmetry property of the transition matrix elements. The total cross section is computed as a sum over the final and average over the initial degenerate states, as usual.

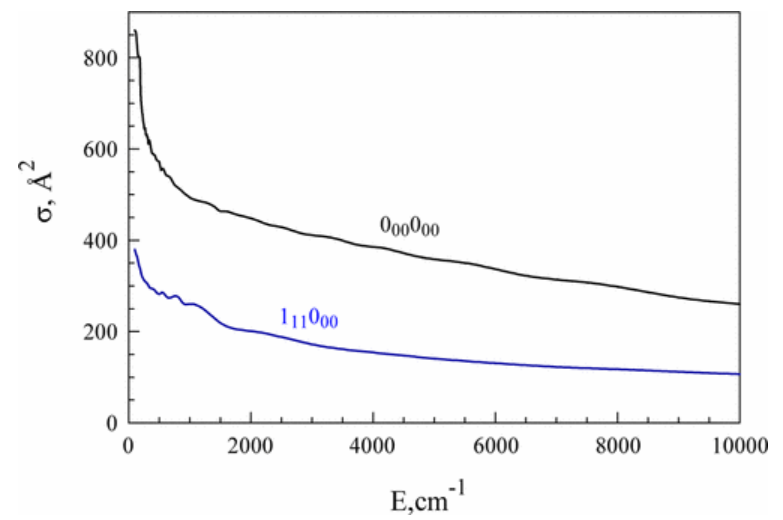

Figure 9. Integral cross section as a function of collision energy for the elastic scattering of the $\mathrm{H}_{2} \mathrm{O}$ $+\mathrm{H}_{2} \mathrm{O}$ system in the ground state $0_{00} 0_{00}$ (black) and in the excited state $1_{11} 0_{00}$ (blue).

Both dependencies in Figure 9 are smooth and exhibit similar behavior: the cross section progressively decreases as the collision energy is raised. At higher energies, the absolute value of the elastic cross section for the excited state $1_{11} 0_{00}$ is about 2 times smaller compared to that of the ground state $0_{00} 0_{00}$. This can be viewed as a symmetry effect. Namely, in contrast to the ground state of the system, $0_{00} 0_{00}$, that has only one component of positive exchange parity, the total wave function for the excited state $1_{11} 0_{00}$ contains two components according to eq 21 . Since transitions between states of opposite exchange parities are forbidden (see eq 26, section II-E), only the states of one parity need to be included in the basis set and treated separately from states of opposite parity. The results presented here were carried out for the positive parity component only (as the initial state). Since for the elastic channel the final state is the same positive parity component of $1_{11} 0_{00}$, such calculations account only for one-half of the (all hypothetically possible) transitions and predict the elastic cross section close to one-half of the total value. Calculations for the negative parity component of the excited state as the initial state would recover another half of the crosssection value.

\section{Conclusions}

In this paper, we worked out the mixed quantum/classical theory for the inelastic collision of two asymmetric-top rotors, which is the most general case of two-body scattering. In this method, the state-to-state transitions between the internal rotational (or ro-vibrational) states of molecules are treated quantum mechanically using the time-dependent Schrodinger equation, whereas their relative translational motion (responsible for scattering) is treated classically using the average trajectory (Ehrenfest) approach. Two versions of the formula for transition matrix elements were presented: a straightforward approach that uses numerical multidimensional quadrature over all internal degrees of freedom and a (more standard) analytic approach that uses an expansion of the PES over the basis set of spherical harmonics. Adaptation to the case of identical-molecule scattering was also presented. 
This theory was then applied to the rotational excitation of two water molecules, $\mathrm{H}_{2} \mathrm{O}+\mathrm{H}_{2} \mathrm{O}$, using the PES from recent literature. Properties of the expansion coefficients of the PES, and of the stateto-state transition matrix elements, were analyzed to reveal the major features of this system, such as a long-range dipole-dipole interaction. Calculations of collisional excitation from the ground state of the system, $0_{00} 0_{00}$, into a number of low-lying excited rotational states were carried out in a broad range of energies, up to $10000 \mathrm{~cm}^{-1}$. Analysis of computed opacity functions showed a rather unusual scattering regime, dominated by a strong anisotropic long-range interaction (dipoledipole). Several most important dipole-driven transitions were identified and discussed in detail. The coupled-states approximation was tested and found to agree semiquantitatively (within a factor of 2) with the fully coupled version of the method. Differential cross sections for the elastic scattering were computed for several collision energies and found to have a very narrow forward scattering peak.

The computer program written for this work is now being packaged into a user-friendly suite and will be made available for the community as a part of a forthcoming publication: Semenov, A.; Babikov, D. "MQCT. II. User-Ready Program for Calculations of Inelastic Scattering of Two Molecules", which we announce here. It is being designed to have an interface similar to that of MOLSCAT, such as simple input files and the same way of feeding into the PES.

\section{Supporting Information}

The Supporting Information is available free of charge on the ACS Publications website at DOI: $10.1021 /$ acs.jpca.7b03554.

- Level and quantum numbers, rotational energies, cross section data, PES expansion terms $(\underline{\text { ZIP }})$

o Zip jip7b03554 si 002.zip (1.26 MB)

\section{Acknowledgment}

This research was supported by the NASA Astrophysics Program, grant no. NNX17AH16G. We used resources of the National Energy Research Scientific Computing Center, which is supported by the Office of Science of the U.S. Department of Energy under Contract no. DE-AC02-5CH11231. Alexander Semenov acknowledges support of the Jobling Research Assistantship at Marquette.

\section{References}

1 Arthurs, A. M.; Dalgarno, A. The Theory of Scattering by a Rigid Rotator Proc. R. Soc. London, Ser. A 1960, 256,540- 551DOI: 10.1098/rspa.1960.0125

$\underline{2}$ Green, S . Rotational Excitation in $\mathrm{H}_{2}-\mathrm{H}_{2}$ Collisions: Close-coupling Calculations. J. Chem. Phys. 1975, 62,2271- 2282;.DOI: 10.1063/1.430752. Green, S. Rotational Excitation of Symmetric Top Molecules by Collisions with Atoms: Close Coupling, Coupled States, and Effective Potential Calculations for $\mathrm{NH}_{3}-$ He. J. Chem. Phys. 1976, 64, 3463- 3473.DOI: $10.1063 / 1.432640$

$\underline{3}$ Hutson, J. M.; Green, S.MOLSCAT Computer Code, version 14; Distributed by Collaborative Computational Project No. 6; Engineering and Physical Sciences Research Council: U.K., 1994. http://www.theochem.ru.nl/molscat/TOC.html. 
4 Alexander, M. H.; Manolopoulos, D. E.; Werner, H.-J.; Follmeg, B.HIBRIDON; available at http://www2.chem.umd.edu/groups/alexander/hibridon/hib43/hibhelp.html.

5 Loreau, J.; Zhang, P.; Dalgarno, A. Elastic scattering and rotational excitation of nitrogen molecules by sodium atoms J. Chem. Phys. 2011, 135, 174301DOI: 10.1063/1.3653983

6 Balakrishnan, N.; Dalgarno, A.; Forrey, R. C. Vibrational relaxation of CO by collisions with ${ }^{4} \mathrm{He}$ at ultracold temperatures J. Chem. Phys. 2000, 113, 621- 627DOI: 10.1063/1.481838

7 Lique, F.; Cernicharo, J.; Cox, P. The Excitation of SO in Cold Molecular Clouds: TMC-1 Astrophys. J. 2006, 653,1342DOI: 10.1086/508978

$\underline{8}$ dos Santos, F. S.; Balakrishnan, N.; Forrey, R. C.; Stancil, P. C. Vibration-vibration and Vibrationtranslation Energy Transfer in $\mathrm{H}_{2}-\mathrm{H}_{2}$ Collisions: a Critical Test of Experiment with FullDimensional Quantum Dynamics J. Chem. Phys. 2013, 138, 104302DOI: $10.1063 / 1.4793472$

9-Ndengue, S. A.; Dawes, R.; Gatti, F. Rotational Excitations in CO-CO Collisions at Low Temperature: Time Independent and Multiconfigurational Time Dependent Hartree Calculations J. Phys. Chem. A 2015, 119, 7712-7723DOI: 10.1021/acs.jpca.5b01022

10 Janssen, L. M. C.; van der Avoird, A.; Groenenboom, G. C. Quantum Reactive Scattering of Ultracold NH(X,3 $\left.{ }^{-}\right)$Radicals in a Magnetic Trap Phys. Rev. Lett. 2013, 110, 063201D0I: 10.1103/PhysRevLett.110.063201

11 Yang, B.; Zhang, P.; Wang, X.; Stancil, P. C.; Bowman, J. M.; Balakrishnan, N.; Forrey, R. C. Quantum Dynamics of CO-H $\mathrm{H}_{2}$ in Full Dimensionality Nat. Commun. 2015, 6, 6629DOI: 10.1038/ncomms7629

12 Yang, B.; Nagao, M.; Satomi, W.; Kimura, M.; Stancil, P. C. Rotational Quenching of Rotationally Excited $\mathrm{H}_{2} \mathrm{O}$ in Collisions with He Astrophys. J. 2013, 765, 77-77DOI: 10.1088/0004637X/765/2/77

13 Daniel, F.; Dubernet, M.-L.; Grosjean, A. Rotational excitation of 45 levels of ortho/para- $\mathrm{H}_{2} \mathrm{O}$ by excited ortho/para- $\mathrm{H}_{2}$ from 5 to $1500 \mathrm{~K}$ : state-to-state, effective, and thermalized rate coefficients Astron. Astrophys.2011, A76, 536- 546DOI: 10.1051/0004-6361/201118049

14 Yang, C. H.; Sarma, G.; Parker, D. H.; ter Meulen, J. J.; Wiesenfeld, L. State-to-state Differential and Relative Integral Cross Sections for Rotationally Inelastic Scattering of $\mathrm{H}_{2} \mathrm{O}$ by Hydrogen J. Chem. Phys. 2011, 134,204308DOI: 10.1063/1.3589360

15 Faure, A.; Wiesenfeld, L.; Scribano, Y.; Ceccarelli Rotational Excitation of Mono- and DoublyDeuterated Water by Hydrogen C Mon. Not. R. Astron. Soc. 2012, 420, 699- 704DOI: 10.1111/j.1365-2966.2011.20081.x

16 Wernli, M.; Wiesenfeld, L.; Faure, A.; Valiron, P. Rotational excitation of $\mathrm{HC}_{3} \mathrm{~N}$ by $\mathrm{H}_{2}$ and $\mathrm{He}$ at low temperatures Astron. Astrophys. 2007, 464, 1147-1154DOI: 10.1051/00046361:20066112

17 Troscompt, N.; Faure, A.; Wiesenfeld, L.; Ceccarelli, C.; Valiron, P. Rotational Excitation of Formaldehyde by Hydrogen Molecules: Ortho- $\mathrm{H}_{2} \mathrm{CO}$ at Low Temperature Astron. Astrophys. 2009, 493, 687- 696DOI: 10.1051/0004-6361:200810712

18 Rabli, D.; Flower, D. R. The Rotational Excitation of Methanol by Molecular Hydrogen Mon. Not. R. Astron. Soc. 2010, 406, 95DOI: 10.1111/j.1365-2966.2010.16671.x

19 Faure, A.; Szalewicz, K.; Wiesenfeld, L. Potential Energy Surface and Rotational Cross Sections for Methyl Formate Colliding with Helium J. Chem. Phys. 2011, 135, 024301D0I: $10.1063 / 1.3607966$ 
20 Roueff, E.; Lique, F. Molecular Excitation in the Interstellar Medium: Recent Advances in Collisional, Radiative, and Chemical Processes Chem. Rev. 2013, 113, 8906- 8938DOI: $10.1021 / \mathrm{cr} 400145 \mathrm{a}$

21 Semenov, A.; Ivanov, M. V.; Babikov, D. Ro-vibrational Quenching of CO ( $\mathrm{v}=1)$ by He Impact in a Broad Range of Temperatures: A Benchmark Study Using Mixed Quantum/Classical Inelastic Scattering Theory J. Chem. Phys.2013, 139, 074306DOI: 10.1063/1.4818488

22 Semenov, A.; Babikov, D. Quantum/Classical Calculations of Total and Differential Elastic and Rotationally Inelastic Scattering Cross Sections for Light and Heavy Reduced Masses in a Broad Range of Collision EnergiesJ. Chem. Phys. 2014, 140 (13) 044306DOI: 10.1063/1.4862409

23 Semenov, A.; Babikov, D. Accurate Calculations of Rotationally Inelastic Scattering Cross Sections Using Mixed Quantum/Classical Theory J. Phys. Chem. Lett. 2014, 5, 275- 278DOI: 10.1021/jz402542w

24 Ivanov, M. V.; Dubernet, M.-L.; Babikov, D. Rotational Quenching of $\mathrm{H}_{2} \mathrm{O}$ by He: Mixed Quantum/Classical Theory and Comparison with Quantum Results J. Chem. Phys. 2014, 140 (7) 134301- 134301DOI: 10.1063/1.4868715

25 Semenov, A.; Dubernet, M.-L.; Babikov, D. Mixed Quantum/Classical Theory for Inelastic Scattering of Asymmetric-Top-Rotor + Atom in The Body-fixed Reference Frame and Application to the $\mathrm{H}_{2} \mathrm{O}+$ He System J. Chem. Phys. 2014, 141 (8) 114304DOI: $10.1063 / 1.4895607$

26 Semenov, A.; Babikov, D. Mixed Quantum/Classical Approach for Description of Molecular Collisions in Astrophysical Environments J. Phys J. Phys. Chem. Lett. 2015, 6, 1854- 1858DOI: 10.1021/acs.jpclett.5b00496

27 Semenov, A.; Babikov, D. Mixed Quantum/Classical Theory for Molecule-Molecule Inelastic Scattering: Derivations of Equations and Application to $\mathrm{N}_{2}+\mathrm{H}_{2}$ System J. Phys. Chem. A 2015, 119, 12329-12338DOI: 10.1021/acs.jpca.5b06812

28 Babikov, D.; Semenov, A. Recent Advances in Development and Applications of the Mixed Quantum/Classical Theory for Inelastic Scattering J. Phys. Chem. A 2016, 120, 319- 331DOI: 10.1021/acs.jpca.5b09569

29 Semenov, A.; Babikov, D. Inelastic Scattering of Identical Molecules within Framework of the Mixed Quantum/Classical Theory: Application to Rotational Excitations in $\mathrm{H}_{2}+\mathrm{H}_{2}$ J. Phys. Chem. A 2016, 120, 3861-3866D0I: 10.1021/acs.jpca.6b04556

30 Agg, P. J.; Clary, D. C. Infinite Order Sudden Calculation of Raman Q-Branch Linewidths for $\mathrm{H}_{2} \mathrm{O}+$ $\mathrm{H}_{2} \mathrm{O}$ J. Chem. Phys. 1991, 95, 1037-1048DOI: 10.1063/1.461131

31 Buffa, G.; Tarrini, O.; Scappini, F.; Cecchi-Pestellini, C. $\mathrm{H}_{2} \mathrm{O}-\mathrm{H}_{2} \mathrm{O}$ Collision Rate Coefficients Astrophys. J., Suppl. Ser. 2000, 128, 597DOI: 10.1086/313389

32 Jehin, E.; Bockelee-Morvan, D.; Dello Russo, N.; Manfroid, J.; Hutsemekers, D.; Kawakita, H.; Kobayashi, H.;Schulz, R.; Smette, A.; StueweA Multi-Wavelength Simultaneous Study of the Composition of the Halley Family Comet 8P/Tuttle Earth, Moon, Planets 2009, 105, 343DOI: 10.1007/s11038-009-9317-8

33 Hartogh, P.; Lis, D. C.; Bockelee-Morvan, D.; de Val-Borro, M.; Biver, N.; Kueppers, M.; Emprechtinger, M.; Bergin, E. A.; Crovisier, J.; Rengel, M. Ocean-like water in the Jupiterfamily comet 103P/Hartley 2 Nature 2011, 478, 218DOI: 10.1038/nature10519

34 Bockelee-Morvan, D.; Biver, N.; Swinyard, B.; de Val-Borro, M.; Crovisier, J.; Hartogh, P.; Lis, D. C.; Moreno, R.;Szutowicz, S.; Lellouch, E.Herschel measurements of the D/H and 160/180 
ratios in water in the Oort-cloud comet C/2009 P1 (Garradd) Astron.

Astrophys. 2012, 544, L15DOI: 10.1051/0004-6361/201219744

$\underline{35}$ van Dishoeck, E. F.; Kristensen, L. E.; Benz, A. O.; Bergin, E. A.; Caselli, P.; Cernicharo, J.; Herpin, F.; Hogerheijde, M. R.; Johnstone, D.; Liseau, R.Water in Star-forming Regions with the Herschel Space Observatory (WISH). I. Overview of Key Program and First Results Publ. Astron. Soc. Pac. 2011, 123, 138DOI: 10.1086/658676

36 Demtröder, W. Molecular Physics; John Wiley and Sons Ltd.: Hoboken, NJ, 2008.

37 Varshalovich, D. A.; Moskalev, A. N.; Khersonskii, V. K. Quantum Theory of Angular Momentum; World Scientific: Singapore, 1988.

38 Mas, E. M.; Szalewicz, K.; Bukowski, E.; Jeziorski, B. Pair potential for water from symmetryadapted perturbation theory J. Chem. Phys. 1997, 107, 4207- 4218D0I: 10.1063/1.474795

39 Jankowski, P.; Murdachaew, R.; Bukowski, O.; Akin-Ojo, O.; Leforestier, C.; Szalewicz, K. Ab Initio Water Pair Potential with Flexible Monomers J. Phys. Chem. A 2015, 119, 2940- 2964DOI: $10.1021 /$ jp512847z

40 Huang, X.; Braams, B. J.; Bowman, J. M. New ab initio potential energy surface and the vibrationrotation-tunneling levels of $\left(\mathrm{H}_{2} \mathrm{O}\right)_{2}$ and $\left(\mathrm{D}_{2} \mathrm{O}\right)_{2} \mathrm{~J}$. Chem. Phys. 2008, 128, 034312DOI: $10.1063 / 1.2822115$

$\underline{41}$ Kristensen, L. E.; van Dishoeck, E. F.; Bergin, E. A.; Visser, R.; Yildiz, U. A.; San Jose-Garcia, I.; Joergensen, J. K.;Herczeg, G. J.; Johnstone, D.; Wampfler, S. F.Water in star-forming regions with Herschel (WISH) II. Evolution of $557 \mathrm{GHz}$ 110-101 emission in low-mass protostars et al Astron. Astrophys. 2012, 542, A8DOI: 10.1051/0004-6361/201118146

42 Ceccarelli, C.; Hollenbach, D. G.; Tielens, A. G. G. M. Far-Infrared Line Emission from Collapsing Protostellar Envelopes Astrophys. J. 1996, 471, 400DOI: 10.1086/177978

43 Kaufman, M. J.; Neufeld, D. A. Far-Infrared Water Emission from Magnetohydrodynamic Shock WavesAstrophys. J. 1996, 456, 611DOI: 10.1086/176683

44 Coutens, A.; Vastel, C.; Caux, E.; Ceccarelli, C.; Bottinelli, S.; Wiesenfeld, L.; Faure, A.; Scribano, Y.; Kahane, C. A study of deuterated water in the low-mass protostar IRAS 162932422 Astron. Astrophys. 2012, 539, A132DOI: 10.1051/0004-6361/201117627

45 Taquet, V.; Peters, P. S.; Kahane, C.; Ceccarelli, C.; Lopez-Sepulcre, A.; Toubin, C.; Duflot, D.; Wiesenfeld, L.Water ice deuteration: a tracer of the chemical history of protostars Astron. Astrophys. 2013, 550, A127DOI: 10.1051/0004-6361/201220084

46 Kirste, M.; Wang, X.; Schewe, H. C.; Meijer, G.; Liu, K.; van der Avoird, A.; Janssen, L. M.; Gubbels, K. B.;Groenenboom, G. C.; van de Meerakker, S. Y. Quantum-State Resolved Bimolecular Collisions of Velocity-Controlled OH with NO Radicals Science 2012, 338, 1060-1063DOI: $10.1126 /$ science. 1229549

$\underline{47}$ Ndengue, S. A.; Dawes, R.; Gatti, F. Rotational Excitations in CO-CO Collisions at Low Temperature: Time-Independent and Multiconfigurational Time-Dependent Hartree Calculations J. Phys. Chem. A 2015, 119, 7712-7723DOI: 10.1021/acs.jpca.5b01022

48 Avdeenkov, A. V.; Bohn, J. L. Collisional dynamics of ultracold OH molecules in an electrostatic field Phys. Rev. A: At., Mol., Opt. Phys. 2002, 66, 052718DOI: 10.1103/PhysRevA.66.052718 